\title{
Jet-hadron correlations measured relative to the second order event plane in Pb-Pb collisions at $\sqrt{s_{N N}}=2.76 \mathrm{TeV}$
}

\author{
S. Acharya et al.* \\ (ALICE Collaboration)
}

(Received 16 November 2019; accepted 10 February 2020; published 3 June 2020)

\begin{abstract}
The quark gluon plasma produced in ultrarelativistic heavy-ion collisions at the Large Hadron Collider (LHC) can be studied by measuring the modifications of jets formed by hard scattered partons which interact with the medium. We studied these modifications via angular correlations of jets with charged hadrons for jets with momenta $20<p_{\mathrm{T}}^{\text {jet }}<40 \mathrm{GeV} / c$ as a function of the associated particle momentum. The reaction plane fit method is used in this analysis to remove the flow modulated background. The analysis of angular correlations for different orientations of the jet relative to the second order event plane allows for the study of the path length dependence of medium modifications to jets. We present the dependence of azimuthal angular correlations of charged hadrons with respect to the angle of the axis of a reconstructed jet relative to the event plane in $\mathrm{Pb}-\mathrm{Pb}$ collisions at $\sqrt{s_{N N}}=2.76 \mathrm{TeV}$. The dependence of particle yields associated with jets on the angle of the jet with respect to the event plane is presented. Correlations at different angles relative to the event plane are compared through ratios and differences of the yield. No dependence of the results on the angle of the jet with respect to the event plane is observed within uncertainties, which is consistent with no significant path length dependence of the medium modifications for this observable.
\end{abstract}

DOI: 10.1103/PhysRevC.101.064901

\section{INTRODUCTION}

A hot, dense liquid of quarks and gluons is created in high energy collisions of heavy ions at the Relativistic Heavy Ion Collider (RHIC) [1-4] and the Large Hadron Collider (LHC) [5-11]. This strongly interacting medium, called the quark gluon plasma (QGP), suppresses colored probes such as quarks and gluons.

Hard parton scatterings occur early in the collision and lead to the production of jets, collimated sprays of particles formed from the fragmentation of the scattered parton. These hard partons lose energy through induced gluon bremsstrahlung and elastic collisions with medium partons as they traverse the QGP, leading to a broadening of the resulting jet and softening of its constituents [12,13]. This energy loss can be studied with measurements of high transverse momentum hadrons or reconstructed jets. High momentum charged hadron production is suppressed by a factor of approximately 5 in Au-Au collisions at RHIC [14-16] and up to a factor of nearly 10 in $\mathrm{Pb}-\mathrm{Pb}$ collisions at the $\mathrm{LHC}[7,17]$ relative to that in $p p$ collisions. These measurements are used to constrain the transport coefficient $\hat{q}$, the squared partonic momentum exchanged with the medium divided by the path length traversed [18] in the QGP.

\footnotetext{
${ }^{*}$ Full author list given at the end of the article.
}

Published by the American Physical Society under the terms of the Creative Commons Attribution 4.0 International license. Further distribution of this work must maintain attribution to the author(s) and the published article's title, journal citation, and DOI.
An enhancement of particle production at low $p_{\mathrm{T}}$ due to medium interactions has been observed with measurements of fragmentation functions, the momentum distributions of particles within the jet [19-21], as well as through broadening in high momentum dihadron correlations [22,23] and jethadron correlations [11,24-26].

Measurements of correlations allow studies of lower energy jets and of the soft constituents by means of statistical subtraction of the large combinatorial background at lower momenta. Studies of correlations have been limited by methods for background subtraction due to the structures in the background correlated with the signal because of hydrodynamical flow. The recent development of the reaction plane fit (RPF) method enables precision subtraction of the background for both jet-hadron and dihadron correlations [27].

The path length dependence of partonic energy loss can be constrained through measurements of the dependence of azimuthal correlations of high momentum particles or reconstructed jets on the angle of the jet relative to second order event plane of the collision. Because the overlap region of the incoming nuclei for non-central collisions is almond shaped, particles traveling perpendicular to this event plane (out-of-plane) have a longer path length through the medium on average than those traveling in the direction of the event plane (in-plane). Therefore, the suppression of high momentum single particles is expected to be greater in the out-ofplane direction than in-plane [28]. This is also evident in the azimuthal anisotropy of high- $p_{\mathrm{T}}$ single particle [29-32] and jet $[33,34]$ production relative to the second order event plane. This suppression indicates that there are fewer jets out-ofplane after interactions with the medium, but is not a measure 
of the properties of the surviving jets relative to that plane. Measurements of dihadron correlations relative to the event plane at RHIC indicate suppression and some broadening [35,36], but do not exhibit much event plane dependence [37]. Some theoretical studies indicate that jet-by-jet fluctuations in the energy loss may be as important as the path length dependence for some observables, such as azimuthal anisotropies at high momentum and dijet asymmetries [38-40].

Measurements for the event plane dependence of jet modification can therefore provide insight into the relative importance of path length for partonic energy loss. We present measurements of jet-hadron correlations relative to the event plane in $\mathrm{Pb}-\mathrm{Pb}$ collisions at $\sqrt{s_{N N}}=2.76 \mathrm{TeV}$ using $\mathrm{A}$ Large Ion Collider Experiment (ALICE) detector. We first describe the details of the measurement technique and then present the results. We conclude with a discussion of the constraints these measurements provide for models.

\section{THE ALICE DETECTOR}

A detailed description of the ALICE detector and its subdetectors can be found in Ref. [41]. The detectors used for the present analysis are briefly described in this section. These are the forward scintillator arrays (V0) $[42,43]$, inner tracking system (ITS) [44], the time projection chamber (TPC) [45], and the electromagnetic calorimeter (EMCal) [46].

The V0 detector is used for centrality estimation and event plane reconstruction. The V0 system consists of two scintillator arrays located at asymmetric positions, one at a pseudorapidity range of $2.8<\eta<5.1$ (V0A) and the other at $-3.7<\eta<-1.7$ (V0C) [42]. Each set of arrays is made of four radial rings with each ring divided into eight sections in the azimuthal direction [42].

The ITS and TPC detectors provide tracking of charged particles over the full range of azimuth with a pseudorapidity range of $|\eta|<0.9$. They are located inside the central barrel solenoidal magnet which provides a homogeneous field with strength of $0.5 \mathrm{~T}$ [47]. The ITS is a cylindrical silicon detector made up of six layers located at the center of the main barrel. The first two layers are the silicon pixel detectors (SPDs), followed by two layers of silicon drift detectors (SDDs), and two outer layers of silicon strip detectors (SSDs) [48]. The TPC surrounds the ITS and is the main detector used for tracking in ALICE. It is filled with a gas mixture of $\mathrm{Ne}$ and $\mathrm{CO}_{2}$ [49]. The transverse momentum and charge of the particles can be inferred from the curvature of the tracks. Combining information from the ITS and TPC allows for the momentum determination of charged particles as low as $p_{\mathrm{T}} \approx$ $0.15 \mathrm{GeV} / c$ up to $p_{\mathrm{T}} \approx 100 \mathrm{GeV} / c$.

Track selection is optimized for track quality, momentum resolution, and nearly uniform azimuthal acceptance, as in [47]. At least three hits in the ITS are required. Tracks without a hit in the SPD are refit to include the primary vertex, reducing the azimuthal dependence of the track reconstruction efficiency while maintaining good momentum resolution. The tracks used in this analysis are required to have $80 \%$ of the geometrically allowed space points and at least 70 total space points in the TPC. The tracking efficiency is determined from simulations of the detector response using tracks simu- lated with HIJING [50] propagated through the detector using GEANT3 [51] and ranges 80-85\% in the momentum range used in this analysis.

The uncertainty on the single track reconstruction efficiency is $4 \%$, with an additional $1 \%$ systematic uncertainty due to contamination from secondary tracks [47,52-54]. This uncertainty is correlated point-to-point and contributes to the scale uncertainty in the correlation functions and yields.

The EMCal is used for the neutral energy reconstruction and triggering. It is a lead-scintillator sampling calorimeter with a pseudorapidity coverage of $|\eta|<0.7$ and an active azimuthal range of $\Delta \phi=107^{\circ}$ in the readout in the 2011 configuration $[46,55,56]$. The EMCal had 11520 towers with transverse size $6 \mathrm{~cm} \times 6 \mathrm{~cm}$, or approximately twice the effective Molière radius. The relative energy resolution is $0.11 / \sqrt{E}+0.017+0.051 / E$, where the energy $E$ is measured in $\mathrm{GeV}$ [55]. Clusters are formed by combining signals from adjacent towers and each cluster is required to have only one local energy maximum. This analysis uses events triggered by a high energy deposit in a $4 \times 4$ region of towers in the EMCal. This trigger configuration has less sensitivity to the underlying event than a trigger configuration with a larger area, often used for jet analyses, because the contribution of the underlying event to the energy is proportional to the trigger area. The raw trigger threshold was multiplicity dependent and corresponded to approximately $4.5-6 \mathrm{GeV}$ in the centrality bin used in this analysis.

Clusters with energy above $3 \mathrm{GeV}$, which exclude minimally ionizing particles [56], are used in this analysis. Partially formed hadronic showers may still pass this threshold. To avoid overcounting of charged particle $p_{\mathrm{T}}$, the cluster energies are corrected as in Ref. [53]. Tracks are propagated to the average cluster depth in the EMCal and matched to the nearest cluster. If the nearest cluster is within $|\Delta \eta|<$ 0.015 in pseudorapidity and $|\Delta \phi|<0.025$ in azimuth, the cluster most likely arose from a charged hadron. If the cluster energy is at or below the track's momentum, the cluster is not used in the analysis, while if the cluster energy is above the track's momentum, the track momentum is subtracted from the cluster energy [53].

\section{METHOD}

The data used in this analysis were collected during the 2011 run [41] from $0.5 \mathrm{M} 30-50 \%$ central $\mathrm{Pb}-\mathrm{Pb}$ collisions at $\sqrt{s_{N N}}=2.76 \mathrm{TeV}$. It was additionally required to be triggered by a high energy deposit in a $4 \times 4$ region of towers in the EMCal [56]. Procedures for selection and reconstruction of tracks from charged particles, identification of calorimeter clusters, and jet reconstruction are as in Ref. [47] and are summarized in Sec. III A. Estimates of the distributions of corrected jet energies are also reported here. The experimentally reconstructed second order symmetry plane is called the second order event plane, referred to as the "event plane" later in the text for simplicity. Centrality determination and event plane reconstruction is discussed in Sec. III B. Jets from triggered events are correlated with all charged tracks in azimuth $\left(\Delta \phi=\phi_{\text {jet }}-\phi_{\text {assoc }}\right)$ and pseudorapidity $\left(\Delta \eta=\eta_{\text {jet }}-\eta_{\text {assoc }}\right)$, as discussed in Sec. III C. The distributions of these associated 


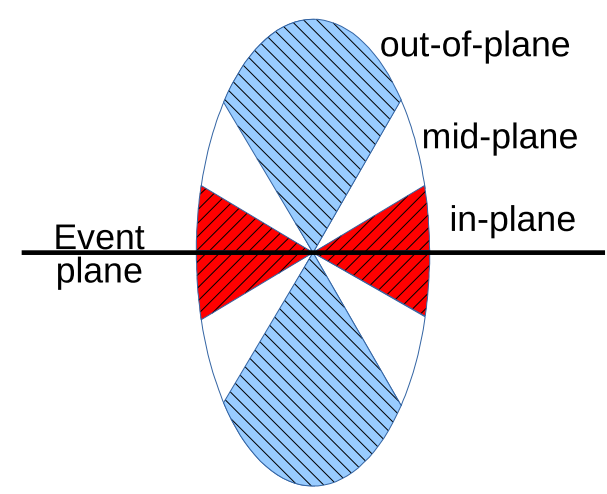

FIG. 1. Jet-hadron correlations are measured for jets in three regions relative to the $n=2$ event plane, which is transverse to the direction of the beams. These regions include in-plane $\left(\left|\psi-\phi_{\text {jet }}\right|<\right.$ $\pi / 6)$ shown in red, mid-plane $\left(\pi / 6<\left|\psi-\phi_{\text {jet }}\right|<\pi / 3\right)$ shown in white, and out-of-plane $\left(\left|\psi-\phi_{\text {jet }}\right|>\pi / 3\right)$ shown in blue.

tracks relative to the trigger jet are measured in three bins in the angle between the trigger jet and the event plane, in-plane $\left(\left|\psi-\phi_{\text {jet }}\right|<\pi / 6\right)$, mid-plane $\left(\pi / 6<\left|\psi-\phi_{\text {jet }}\right|<\right.$ $\pi / 3)$, and out-of-plane $\left(\left|\psi-\phi_{\text {jet }}\right|>\pi / 3\right)$ bins, as shown in Fig. 1. The analysis is restricted to $30-50 \%$ central $\mathrm{Pb}-\mathrm{Pb}$ collisions because this is where the event plane resolution is highest and therefore the analysis will be most sensitive to any path length dependencies. The subtraction of the combinatorial background using the RPF method [27] is discussed in Sec. III D. The determination of the yields and the widths is discussed in Sec. III E. The possible impact of the finite event plane resolution on the signal is discussed in Sec. III F.

\section{A. Jet reconstruction and energy distribution}

Tracks and corrected EMCal clusters are clustered into jets using the anti- $k_{T}$ algorithm with a resolution parameter $R=0.2$ in the FastJet package [57]. Jet transverse momenta are calculated as the scalar sum of their constituent transverse momenta using a boost-invariant $p_{\mathrm{T}}$ recombination scheme. Tracks are assumed to be pions and clusters to arise from massless particles. In order to suppress contributions from combinatorial jets and the contribution of uncorrelated background to the jet energy, jets are reconstructed with constituents above $p_{\mathrm{T}}>3 \mathrm{GeV} / c$ and are required to have an area of at least 0.08 calculated using ghost particles as described in Ref. [57]. Jets are required to be within $\left|\eta_{\text {jet }}\right|<0.5$ and $1.6<\phi_{\text {jet }}<2.9$ so that the entire jet is contained within the EMCal acceptance.

Small jets are used to reduce the impact of the background, as background contributions scale with $R^{2}$. Additionally, to further suppress contributions of the background to the energy and to match trigger conditions [46], the jets are required to contain a cluster with transverse energy larger than $6 \mathrm{GeV}$. We note that this requirement leads to a selection of biased jets, explicitly biasing the near side. The away side, in contrast, is not explicitly biased, although it is unlikely to be a random sample of the jet population. With these constituent cuts, the background contribution to the energy is negligible using estimates of the background per unit area as in Ref. [58]. The background contribution to the energy is therefore not subtracted from the jets, although any residual contribution would be included in the energy distribution estimation. The jet energy is not corrected for detector effects, but the distribution of particle level jet energies in the sample is estimated using PYTHIA6 [59] Tune A [60] simulations embedded at detector level into data measured in $\mathrm{Pb}-\mathrm{Pb}$ collisions and matched back to generator level.

Detector effects such as the single track reconstruction efficiency, momentum resolution in the tracking detectors, and energy resolution in the calorimeter combined with contributions from particles which are not directly observed, such as neutrons and $K_{\mathrm{L}}^{0}$, and contributions from the background lead to a finite energy resolution. This means that when jethadron correlations are measured at a particular jet $p_{\mathrm{T}}$, the distribution of true jet momenta is broad. A full correction for this effect would require two dimensional unfolding with jet-hadron correlations measured for several jet momenta. The current statistics do not allow for such measurements. Instead, we estimate the distribution of true jet energies and focus on comparisons between jets at different angles relative to the event plane to enable the highest precision search for path length dependence allowed by the currently available data.

The kinematic selection of tracks and clusters used in jet finding is chosen to suppress contributions from the combinatorial background and reduce smearing of the jet energy due to the large combinatorial background. With these kinematic selections, the background density is negligible and showed no event plane dependence. Given that no event plane dependence is observed in the signal, this also means that the resolution of the jet axis does not vary with the angle of the jet relative to the event plane.

PYTHIA6 TuneA $[59,60]$ simulations of $p p$ collisions with jets are embedded at detector level into $30-50 \% \mathrm{~Pb}-\mathrm{Pb}$ data. The embedded events are analyzed with the same parameters and cuts as the data analysis, including the jet constituent and cluster biases, while the generator level jets are measured for $p_{\mathrm{T}}>5 \mathrm{GeV} / c$. The generator level jets are first matched geometrically to PYTHIA detector level jets, and then the detector level jets are geometrically matched to jets found within $R=$ 0.2 in the embedded event, with the additional requirement the associated generator level jet distribution is measured. Each such distribution is normalized within the region $20 \leqslant p_{\mathrm{T}}<$ $100 \mathrm{GeV} / c$ where fluctuations are minimized and, assuming that the jet energy distribution in the data is the same as that provided by PYTHIA6, describes the generator level jet distribution that corresponds to the measured detector level jet distribution, shown in Fig. 2. The uncertainty on the jet energy scale is $2.6 \%$ and the jet energy resolution, which is also encoded in the response matrix, is around $20 \%$ for the jets selected in this analysis [53]. There are slight differences in the jets reconstructed with $p_{\mathrm{T}} \leqslant 20 \mathrm{GeV} / c$ for jets at different angles relative to the event plane due to a low momentum embedded jet overlapping with a another jet in the $\mathrm{Pb}-\mathrm{Pb}$ data. Since there are more jets in-plane than out-of-plane in the data, this leads to an apparent difference in the reconstructed jet spectra. Otherwise there are no significant differences between jets at different angles relative to the event plane. 


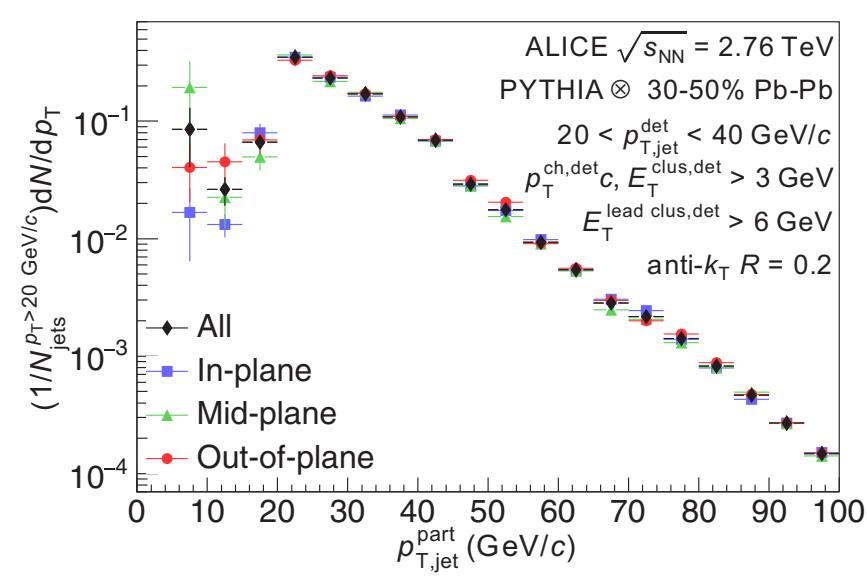

FIG. 2. The generator level jet probability distribution corresponding to jets measured with $p_{\mathrm{T}}$ between 20 and $40 \mathrm{GeV} / c$ for PYTHIA events embedded in $30-50 \%$ central $\mathrm{Pb}-\mathrm{Pb}$ collisions. Generator level jets are required to have $p_{\mathrm{T}}>5 \mathrm{GeV} / c$. The distribution is measured for each angle relative to the event plane, as well as the sum of all angles.

\section{B. Centrality determination and event plane reconstruction}

Centrality is determined from the sum of the energy deposition [42] in the V0 scintillator tiles, as described in Ref. [61]. The centrality of the collision is reported as percent ranges of the total hadronic cross section, with lower percentiles referring to the most central (largest multiplicity) events [61]. The second order event plane $\Psi_{\mathrm{EP}, 2}$ is reconstructed using the V0 following the procedure in [33] by combining signals from the V0A and VOC arrays [43]. The separation in pseudorapidity between the measurement of the signal and the measurement of the event plane suppresses the contribution from the jet signal to the event plane determination [33].

The reaction plane is defined by the beam axis and the vector between the centers of the two incoming nuclei. Additional asymmetries in the distribution of nucleons within the overlap region, generally quantified by a harmonic decomposition, generate symmetry planes at all orders $(n>0)[62,63]$. If the nucleons were in their average positions and there were no fluctuations in interactions between nucleons, the reaction plane would correspond to the second order symmetry plane, $\Psi_{\mathrm{EP}, 2}$. The experimentally reconstructed symmetry planes are called event planes. For simplicity, we refer here to the experimentally reconstructed second order event plane as the event plane. As explained below, we do not correct the signal for the difference between the event and symmetry planes because no event plane dependence is observed in this measurement. Corrections for the event plane resolution will increase differences between results at different angles relative to the event plane but will not induce any event plane dependence if there is none in the uncorrected results. We discuss the impact of the event plane resolution in Sec. III F. The impact of the dijets on the event plane reconstruction was studied in Ref. [33] and found to be negligible.

The event plane is also extracted using TPC tracks in order to determine the event plane resolution. The $n$th order event plane can be calculated from the charged particle azimuthal distribution by [64]

$$
\Psi_{n, \mathrm{EP}}=\left(\arctan \frac{\sum_{i} w_{i} \sin \left(n \phi_{i}\right)}{\sum_{i} w_{i} \cos \left(n \phi_{i}\right)}\right) / n,
$$

where the sum is over all particles in the event, $\phi_{i}$ is the azimuthal angle of the $i$ th particle, and $w_{i}$ is the weight of the $i$ th particle. For measurement of the event plane with the V0, the sum is over all of its sectors and the weights are equal to the amplitude of the respective sector in the $\mathrm{V} 0$, which is proportional to the local multiplicity. A calibration and recentering procedure following [33] is applied to remove any bias introduced by nonuniform acceptance of the V0 system. For measurement in the TPC, tracks are given equal weights $\left(w_{i}=1\right)$ and the acceptance is nearly uniform. Track selection is the same as that described in Sec. III except the transverse momentum range is restricted to $0.15<p_{\mathrm{T}}<5.0 \mathrm{GeV} / c$. More details of the procedure can be found in Ref. [33].

Due to the finite multiplicity of each event, there will be a difference between the symmetry plane and the reconstructed event plane. This difference is quantified by the event plane resolution

$$
\mathfrak{R}_{n}=\left\langle\cos \left(n\left[\Psi_{\mathrm{n}, \mathrm{EP}}-\Psi_{n}\right]\right)\right\rangle,
$$

where $\Psi_{n}$ is the true angle and $\Psi_{n \text {,EP }}$ is the measured angle of the $n$th order event plane. To evaluate the event plane resolution directly from data, this analysis uses three subevents, comparing the event planes measured in the full $\mathrm{V} 0$, measured in the TPC using tracks at positive pseudorapidities, and measured in the TPC using tracks at negative pseudorapidities. We can express the $n$th order resolution of the full $\mathrm{V} 0, \mathfrak{R}_{n}^{V 0}$ $[64,65]$, of the second order event plane by

$$
\mathfrak{R}_{n}^{\mathrm{V} 0}=\left\langle\cos \left(n\left[\Psi_{2, \mathrm{EP}}^{\mathrm{V} 0}-\Psi_{2}\right]\right)\right\rangle=\sqrt{\frac{\left\langle\cos \left(n\left[\Psi_{2, \mathrm{EP}}^{V 0}-\Psi_{2, \mathrm{EP}}^{\mathrm{TPC}, \eta>0}\right]\right)\right\rangle\left\langle\cos \left(n\left[\Psi_{2, \mathrm{EP}}^{\mathrm{V} 0}-\Psi_{2, \mathrm{EP}}^{\mathrm{TPC}, \eta<0}\right]\right)\right\rangle}{\left\langle\cos \left(n\left[\Psi_{2, \mathrm{EP}}^{\mathrm{TP}, \eta>0}-\Psi_{2, \mathrm{EP}}^{\mathrm{TPC}, \eta<0}\right]\right)\right\rangle}},
$$

where $\Psi_{2, \mathrm{EP}}^{V 0}, \Psi_{2, \mathrm{EP}}^{\mathrm{TPC}, \eta>0}$, and $\Psi_{2, \mathrm{EP}}^{\mathrm{TPC}, \eta<0}$ are the second order event planes calculated using the three different subevents, and $\Psi_{2}$ is the true angle of the second order symmetry plane. Fits to the Fourier decomposition of the correlated background are performed up to $n=4$ and are measured relative to the event plane. Thus, event plane resolution corrections $\mathfrak{R}_{2}\left(\psi_{2}\right)$ and $\mathfrak{R}_{4}\left(\psi_{2}\right)$ are needed to correct these terms for the finite precision of the second order event plane measured in the V0 system, as discussed in Sec. IIID. The event plane resolution for the $30-40 \%$ and $40-50 \%$ centrality ranges are combined by weighting the two samples by the number of corresponding events of each. The event plane resolutions $\mathfrak{R}_{2}\left(\psi_{2}\right)$ and $\mathfrak{R}_{4}\left(\psi_{2}\right)$ for the analyzed $30-50 \%$ event sample are 0.73 and 0.44 , respectively, with negligible uncertainties. 


\section{Jet-hadron correlations}

The distribution of charged particles relative to $20<p_{\mathrm{T}}^{\text {jet }}<$ $40 \mathrm{GeV} / c$ reconstructed jets is measured in azimuth $(\Delta \phi)$ and pseudorapidity $(\Delta \eta)$ as

$$
\frac{1}{N_{\text {trig }}} \frac{d^{2} N_{\text {assoc }}}{d \Delta \phi d \Delta \eta}=\frac{1}{N_{\text {trig }}} \frac{1}{a(\Delta \phi, \Delta \eta) \epsilon\left(p_{\mathrm{T}}^{\text {assoc }}, \eta^{\text {assoc }}\right)} \frac{d^{2} N_{\text {meas }}}{d \Delta \phi d \Delta \eta},
$$

where $N_{\text {trig }}$ is the number of trigger jets, $\epsilon\left(p_{\mathrm{T}}^{\text {assoc }}, \eta^{\text {assoc }}\right)$ is the product of the single track reconstruction efficiency and acceptance, and $a(\Delta \phi, \Delta \eta)$ dominantly corrects for the pair acceptance. The distributions are determined in bins of centrality, associated hadron transverse momentum $\left(p_{\mathrm{T}}^{\text {assoc }}\right)$, and bins of the trigger jet angle relative to the event plane.

The correction $a(\Delta \phi, \Delta \eta)$ is calculated as a function of centrality and associated particle momentum by mixed events using a trigger jet from an EMCal-triggered event and associated hadrons from minimum bias events or semicentral triggered events. The mixed event procedure will also remove the trivial correlation due to an $\eta$ dependence in the single particle and track distributions. However, since there is little $\eta$ dependence in either tracks or jets within the acceptance used in this analysis, the dominant effect is the pair acceptance. Mixed events are constructed separately for 30-40\% and $40-50 \%$ centrality classes. The mixed events are required to be within the same $10 \%$ centrality class and have vertex positions within $2 \mathrm{~cm}$ along the direction of the beam, $z_{\mathrm{vtx}}$. There is no difference in the correction within uncertainties for different orientations of the jet relative to the event plane, and therefore the same correction $a(\Delta \phi, \Delta \eta)$ is applied for all angles relative to the event plane. All associated momentum bins for $p_{\mathrm{T}}>2.0 \mathrm{GeV} / c$ are combined to increase statistics because $a(\Delta \phi, \Delta \eta)$ has little momentum dependence at high momenta. The correction $a(\Delta \phi, \Delta \eta)$ is normalized to 1 at its maximum with the systematic uncertainty in the normalization determined by using different regions in $\Delta \phi$ and $\Delta \eta$, with a systematic uncertainty below $0.5 \%$ for all $p_{\mathrm{T}}^{\text {assoc }}$ bins used in this analysis. There is an additional shape uncertainty due to slight changes in the correlation function at large $\Delta \eta$ in the acceptance with $z_{\mathrm{vtx}}$ position. Since the background level is determined from the level of the correlation function at large $\Delta \eta$, this leads to a scale uncertainty in the background subtraction. This uncertainty is determined by varying the binning of the mixed events in $z_{\mathrm{vtx}}$ and is correlated for different angles relative to the event plane and for different bins in $p_{\mathrm{T}}^{\mathrm{assoc}}$. This scale uncertainty is used later for determining a systematic uncertainty on the background subtraction and is dependent on $p_{\mathrm{T}}^{\text {assoc }}$.

\section{Background subtraction}

The signal in Eq. (4) has a large combinatorial background from particles created by processes other than the hard process which created the jet. The jet signal may be correlated with the second order event plane because of jet quenching, and soft hadrons are correlated with the second order event plane due to hydrodynamical flow. The Fourier expansion of this background can be expressed by

$$
\frac{d N}{\pi d \Delta \phi}=B\left(1+\sum_{n=1}^{\infty} 2 \tilde{v}_{n}^{\text {trig }} \tilde{v}_{n}^{\text {assoc }} \cos (n \Delta \phi)\right),
$$

where $\tilde{v}_{n}^{\text {trig }}$ and $\tilde{v}_{n}^{\text {assoc }}$ refer to the effective Fourier coefficients for the azimuthal anisotropy of the trigger jet and associated hadron, respectively, to the background. For inclusive measurements, if the background is dominantly due to flow, the $\tilde{v}_{n}$ of this background will be equal to the $v_{n}$ due to flow. The exact values may be slightly different due to differences in the event samples, varying sensitivity in the method to fluctuations in the $v_{n}$ and nonflow, the difference between the average over all pairs $\left\langle\tilde{v}_{n}^{\text {trig }} \tilde{v}_{n}^{\text {assoc }}\right\rangle$ and the product of the averages over all events $\left\langle\tilde{v}_{n}^{\text {trig }}\right\rangle\left\langle\tilde{v}_{n}^{\text {assoc }}\right\rangle$, differences in the $v_{n}$ for particles in jets and from the bulk, and decorrelations between symmetry planes for hard and soft processes.

The contribution from these soft processes is subtracted using the RPF method [27]. This method avoids contamination by the near- and away-side jets by focusing on the near side only at large $\Delta \eta$ and instead using the dependence of the flow-modulated background on the angle of the trigger jet relative to the event plane to constrain the background shape and level. For in-plane jets, background particles are more likely to be near the trigger jet than $\pi$ away in azimuth, leading to a higher $\cos (2 \Delta \phi)$ term, and the background level is higher because there are more jets in-plane. For out-of-plane jets, background particles are less likely to be near the trigger jet, leading to a negative $\cos (2 \Delta \phi)$ term, and the background is lower because there are fewer jets. Because the second and fourth order event planes are correlated, a similar argument holds for the fourth order terms. These effects help constrain the even $n$ terms and help distinguish them from the odd $n$ terms and constrain the background level while avoiding contamination from the near- and away-side jets.

The event plane dependence can be used to determine the background shape and level. When the angle of the jet is fixed relative to the event plane, the effective size and shape of the background is given by

$$
\begin{aligned}
\tilde{B} & =\frac{N_{t} N_{a} c}{\pi^{2}}\left(1+2 \sum_{k=1}^{\infty} \frac{v_{2 k}^{\mathrm{trig}}}{2 k c} \sin (2 k c) \Re_{2 k} C_{2 k, 0} \cos \left(2 k \phi_{s}\right)\right), \\
\tilde{v}_{n}^{\text {trig }} & =\frac{v_{n}+\frac{\delta_{n, \text { mult } 2}}{n c} \sin (n c) \Re_{n} C_{n, 0} \cos \left(n \phi_{s}\right)+\sum_{k=1}^{\infty}\left(v_{2 k+n}^{\text {trig }} C_{|2 k+n|, n}+v_{|2 k-n|}^{\text {trig }} C_{|2 k-n|, n}\right) \frac{\sin (2 k c) \cos \left(2 k \phi_{s}\right) \Re_{2 k}}{2 k c}}{1+2 \sum_{k=1}^{\infty} \frac{v_{2 k}^{\text {trig }}}{2 k c} \sin (n c) \Re_{2 k} C_{2 k, 0} \cos \left(2 k \phi_{s}\right)}, \\
C_{n, m} & =\left\langle\cos \left(n \psi_{n}+m \psi_{m}-(n+m) \psi_{2}\right)\right\rangle
\end{aligned}
$$


where $\phi_{s}$ is the center of the azimuthal range of the trigger particle relative to the event plane, $c$ is the width of that range, $N_{t}$ is the number of triggers, $N_{a}$ is the number of associated particles, $v_{n}^{\text {assoc }}$ are the $v_{n}$ of the associated particles, $v_{n}^{\text {trig }}$ are the $v_{n}$ of the triggers, and $\mathfrak{R}_{n}$ is the event plane resolution given in Eq. (3) [66,67]. Terms $v_{n}$ with $n<1$ are zero. The $C_{n, m}$ terms are a measure of how correlated event planes of different orders are with the second order event plane and are approximately zero when either $n$ or $m$ is odd. This is consistent with the weak correlation between the $n=2$ participant plane and odd order participant planes [68] because the odd $\tilde{v}_{n}$ arise mainly due to fluctuations in the initial state. In this case, the even $\tilde{v}_{n}$ will change when the angle of the jet relative to the event plane is changed while the odd $\tilde{v}_{n}$ remain constant. The equation is expanded to include terms up to $v_{4}$. The term $C_{2,0}=1$ and the terms $C_{4,0}$ and $C_{4,2}$ are approximated to be 1 . The latter assumption will lead to an inconsistency between the $v_{4}$ from independent measurements and from the fit, but the fit will still provide a valid description of the background.

The shape of the background depends on the $\mathfrak{R}_{n}$, which are fixed at the measured values. The fourth order event plane resolution is calculated relative to the second order event plane, consistent with the shape described in Eq. (6). The uncertainties on the event plane resolution are negligible relative to the statistical and background fit uncertainties of the final results.

The jet signal in Eq. (4) can be decomposed into a near side and an away side. The near side is a peak which is narrow in both $\Delta \phi$ and $\Delta \eta$, meaning that it is negligible at large $\Delta \eta$, while the away side is narrow in $\Delta \phi$ but broad in $\Delta \eta$. The correlation function at large $\Delta \eta(0.8<|\Delta \eta|<$ $1.2)$ and small $\Delta \phi(|\Delta \phi|<\pi / 2)$ is fit simultaneously for $\tilde{v}_{n}$ up to $n=4$ for trigger jets in-plane $\left(\left|\psi-\phi_{\text {jet }}\right|<\pi / 6\right)$, mid-plane $\left(\pi / 6<\left|\psi-\phi_{\text {jet }}\right|<\pi / 3\right)$, and out-of-plane $(\mid \psi-$ $\phi_{\text {jet }} \mid>\pi / 3$ ), shown in Fig. 1, to determine the background shape and level. Because the even $\tilde{v}_{n}$ depend on the angle of the jet relative to the event plane, as shown in Eq. (6), the $\tilde{v}_{2}$ and $\tilde{v}_{4}$ of both the trigger jet and the associated particle are determined in the fit while only the product $\tilde{v}_{3}^{\text {jet }} \widetilde{v}_{3}^{\text {assoc }}$ is extracted from the fit. A rapidity-even $\tilde{v}_{1}$ term can arise due to both momentum conservation and fluctuations in the initial state. This rapidity-even $\tilde{v}_{1}$ has been measured for single hadrons and is comparable in magnitude to $\tilde{v}_{2}$ and $\tilde{v}_{3}[69,70]$. This term does not change when the angle of the trigger jet is varied relative to the event plane so the product $\tilde{v}_{1}^{\text {jet }} \tilde{v}_{1}^{\text {assoc }}$ contributes to the background. When the fit function is varied to include this $n=1$ term, it is zero within uncertainties and did not lead to significant differences in the correlation function. Since $\tilde{v}_{1}^{\text {assoc }}$ is known to be nonzero, this likely means that $\tilde{v}_{1}^{\text {jet }}$ is near zero. For associated particles above $p_{\mathrm{T}}>2 \mathrm{GeV} / c$, the background is low and the statistics for the region which is background-dominated on the near side are therefore also low, so the fit is restricted up to $n=3$. The fits used in this analysis therefore have six parameters below $2 \mathrm{GeV} / c, B, \tilde{v}_{2}^{\text {jet }}, \tilde{v}_{2}^{\text {assoc }}, \tilde{v}_{3}^{\text {jet }} \tilde{v}_{3}^{\text {assoc }}, \tilde{v}_{4}^{\text {jet }}$, and $\tilde{v}_{4}^{\text {assoc }}$, and four above. The event plane resolution is fixed and variations within the uncertainties lead to negligible differences in the correlation functions.
Figure 3 shows a sample correlation function in the region which is background-dominated on the near side compared to the fit for in-plane (a), mid-plane (b), and out-of-plane (c) jets and all jets combined (d) for associated particles with momenta $1.5<p_{\mathrm{T}}^{\text {assoc }}<2.0 \mathrm{GeV} / c$. Correlation functions for other $p_{\mathrm{T}}^{\text {assoc }}$ ranges used in this analysis are given in Ref. [71]. The ratio of the difference between the data and the fit to the fit is shown in Figs. 3(e)-3(h), showing that the fit describes the data well. The background subtracted correlation functions in the region $|\Delta \eta|<0.6$ are shown in Figs. 3(i)-3(1). The uncertainties from the background subtraction are propagated using the covariance matrix from the fit. These uncertainties are nontrivially correlated point-to-point and between different bins relative to the event plane and are shown as a blue band. The scale uncertainties on the background are shown as a green band and are correlated point-to-point and between different bins relative to the event plane. The uncertainties due to the single track reconstruction efficiency, contamination, and the normalization of the acceptance correction are uncorrelated with each other but correlated for all points. These uncertainties are combined and listed as the scale uncertainty. The $\tilde{v}_{2}^{\text {jet }}$ and $\tilde{v}_{2}^{\text {assoc }}$ extracted from the fits are in agreement with other ALICE results [30,33].

Finite event plane resolution reduces the event plane dependence of the signal because the measurement in one bin relative to the event plane will have contributions from other bins as well. Techniques for correcting for this effect increase the event plane dependence observed in the uncorrected data [72], but if there is no event plane dependence in the uncorrected data, these corrections will not reveal an event plane dependence. Since no event plane dependence is observed within uncertainties and corrections would increase the complexity of the measurement and the systematic uncertainties, no correction is applied for the finite event plane resolution. The impact of the finite event plane resolution is discussed in Sec. III F.

\section{E. Associated track yields and peak widths}

The yield of tracks associated with jets is calculated by integrating the associated yield:

$$
Y=\frac{1}{N_{\text {trig }}} \int_{\eta_{1}}^{\eta_{2}} \int_{\phi_{1}}^{\phi_{2}} \frac{d\left(N_{\text {meas }}-N_{\text {bkgd }}\right)}{d \Delta \phi} d \Delta \phi d \Delta \eta .
$$

The integration limits of $\phi_{1}=-\pi / 3$ and $\phi_{2}=\pi / 3$ for the near side, $\phi_{1}=2 \pi / 3$ and $\phi_{2}=4 \pi / 3$ for the away side, and $\eta_{1}=-0.6$ and $\eta_{2}=0.6$ for both are part of the definition of the measurement. The systematic uncertainties due to the extraction of the background from the RPF are propagated using the covariance matrix from the fit. This uncertainty is nontrivially correlated between yields for different angles of the jet relative to the event plane and for the near and away sides and uncorrelated for points at different $p_{\mathrm{T}}^{\text {assoc }}$. The shape uncertainty in the acceptance correction at large $\Delta \eta$ leads to an additional scale uncertainty when propagating the background determined in the region $0.8<|\Delta \eta|<1.2$ to the region $(|\Delta \eta|<0.6)$. This uncertainty is $100 \%$ correlated for all data points. The single track reconstruction efficiency uncertainty, the uncertainty due to normalization of the 


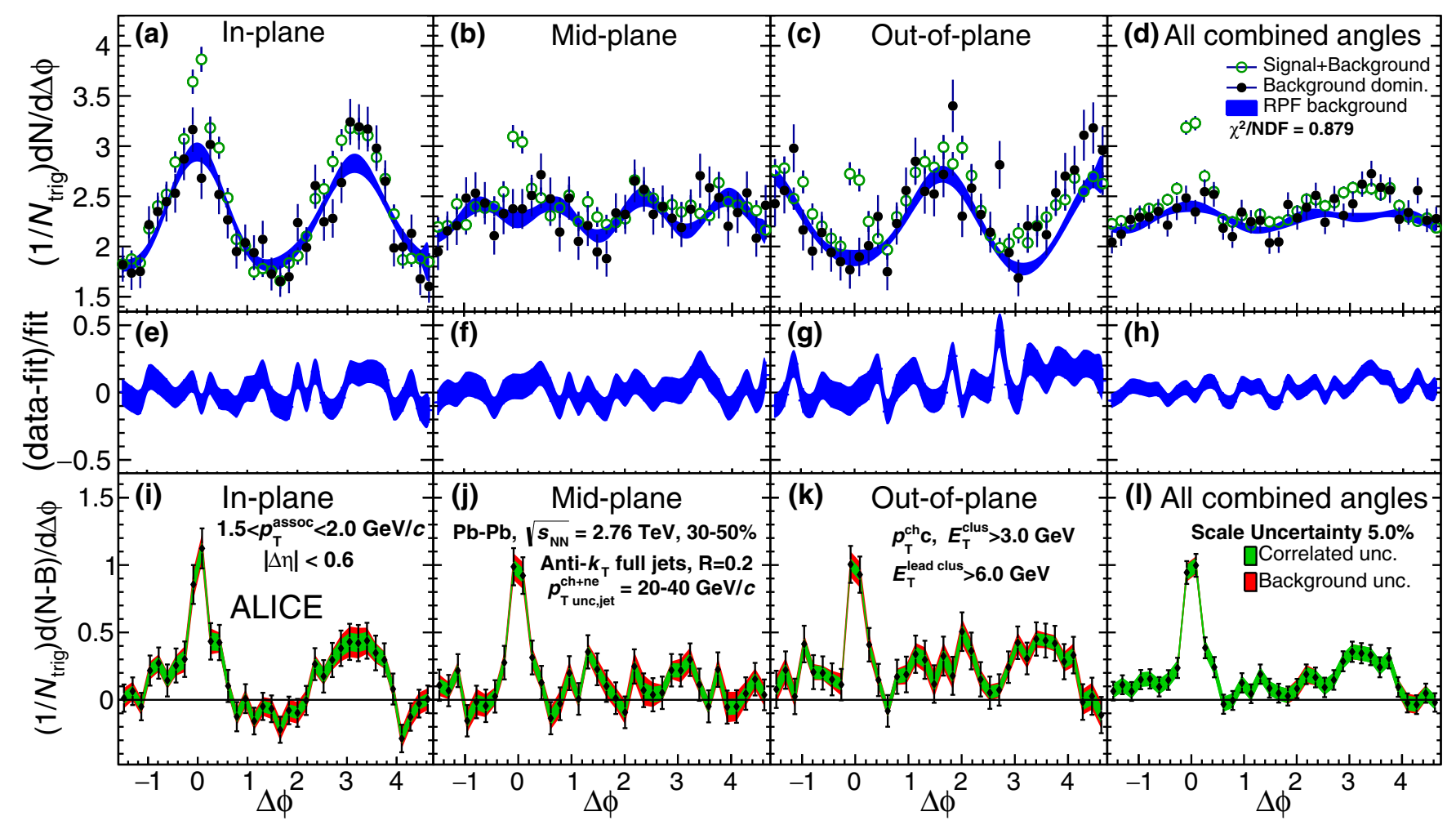

FIG. 3. The signal plus background region, $|\Delta \eta|<0.6$ (green points), the region which is background-dominated on the near side, $0.8<$ $|\Delta \eta|<1.2$ (black points), and the RPF fit to $|\Delta \phi|<\pi / 2$ (blue band) to the region which is background-dominated on the near side for $20<p_{\mathrm{T}}^{\text {jet }}<40 \mathrm{GeV} / c$ jets correlated with $1.5<p_{\mathrm{T}}^{\text {assoc }}<2.0 \mathrm{GeV} / c$ hadrons from $30 \%$ to $50 \%$ centrality collisions on the top panel. The middle panel shows the quality of the RPF fit to the region which is background-dominated on the near side, (data-fit)/fit. On the bottom panel are the RPF corrected correlation functions, with the uncertainty from the background fit (red band), and the correlated uncertainty (green band).

acceptance correction, and the uncertainty due to contamination from secondary particles are $100 \%$ correlated for all points and affect the scale of the correlation functions and the yields.

The ratios and differences of yields are calculated in order to investigate possible event plane dependent modifications. The systematic uncertainties from the background largely cancel out in the ratio and the difference. The track reconstruction efficiency, mixed event normalization, and secondary contamination systematic uncertainties cancel out in the ratio.

The widths are quantified by fitting the correlation functions to a Gaussian, $A e^{\left(\Delta \phi-\Delta \phi_{0}\right)^{2} / 2 \sigma^{2}}$ where $\Delta \phi_{0}=0$ on the near side and $\Delta \phi_{0}=\pi$ on the away side, in the range $|\Delta \phi|<$ $\pi / 3$ on the near side and $|\Delta \phi-\pi|<\pi / 3$ on the away side. The near and away sides are fit separately. The Gaussian fit is repeated with different values of the background parameters and the covariance matrix is used to propagate the uncertainties.

The systematic uncertainties are summarized in Tables I and II. Table I lists the sources of systematic uncertainties which are independent of the angle relative to the event plane and the momentum, including the single track reconstruction efficiency (Sec. II), contamination from secondaries (Sec. II), uncertainties in the mixed events due to their normalization and shape in $\Delta \phi$ (Sec. III C), and uncertainties in the event plane resolution (Sec. III B). The uncertainties in the single track reconstruction efficiency, normalization of the acceptance correction determined from mixed events, and secondary contamination lead to a $5 \%$ uncertainty in the scale of the correlation functions and yields. This uncertainty is uncorrelated for different associated particle momenta.

Table II lists uncertainties which are dependent on the angle of the jet relative to the event plane and the associated particle's momentum on the yields due to the scale uncertainty in the mixed events (Sec. III C) and in the background fit (Sec. IIID) for a few representative associated particle momenta. The uncertainty of the acceptance correction determined from mixed events in $\Delta \eta$ and the uncertainty due to the background subtraction are different for different $p_{\mathrm{T}}^{\text {assoc }}$ bins and therefore shown separately for each data point. The

TABLE I. Summary of systematic uncertainties which are independent of the angle relative to the event plane and the momentum for $20<p_{\mathrm{T}}^{\text {jet }}<40 \mathrm{GeV} / c$ in $30-50 \%$ central $\mathrm{Pb}-\mathrm{Pb}$ collisions.

\begin{tabular}{lc}
\hline \hline Source & Uncertainty $\%$ \\
\hline Single particle reconstruction efficiency & 4 \\
Contamination & 1 \\
Mixed event (shape $\Delta \phi)$ & negligible \\
Mixed event normalization & $<0.5$ \\
Event plane resolution & negligible
\end{tabular}


TABLE II. Summary of systematic uncertainties on the yields and widths calculated from the correlation functions due to the shape uncertainty coming from the shape of the acceptance correction in $\Delta \eta$ and the correlated background fit uncertainty, both varying with event plane orientation bins. They are displayed for $20<p_{\mathrm{T}}^{\text {jet }}<40 \mathrm{GeV} / c$ in $30-50 \%$ central Pb-Pb collisions for $1.0<p_{\mathrm{T}}^{\text {assoc }}<1.5 \mathrm{GeV} / c$ and $3.0<p_{\mathrm{T}}^{\text {assoc }}<4.0 \mathrm{GeV} / c$ bins. The values are expressed as a percent of the nominal value.

\begin{tabular}{|c|c|c|c|c|c|c|}
\hline \multirow[b]{2}{*}{ Source } & \multirow[b]{2}{*}{ Result } & \multirow[b]{2}{*}{ Orientation } & \multicolumn{4}{|c|}{ Uncertainty \% } \\
\hline & & & $1.0-1.5$ & $3.0-4.0$ & $1.0-1.5$ & $3.0-4.0$ \\
\hline \multirow{4}{*}{ Acceptance shape } & \multirow{3}{*}{ Yield } & in-plane & 20 & 2.8 & 33 & 7.9 \\
\hline & & mid-plane & 13 & 2.7 & 25 & 9.2 \\
\hline & & out-of-plane & 10 & 2.5 & 22 & 6.3 \\
\hline & \multirow[t]{2}{*}{ Width } & mid-plane & 9.8 & 1.4 & & 7.1 \\
\hline \multirow{5}{*}{ Background fit } & & out-of-plane & 5.9 & 0.9 & & 4.6 \\
\hline & \multirow{3}{*}{ Yield } & in-plane & 16 & 6.3 & 50 & 18 \\
\hline & & mid-plane & 9.3 & 3.9 & 37 & 13 \\
\hline & & out-of-plane & 7.9 & 6.0 & 35 & 15 \\
\hline & Width & in-plane & 23 & 4.2 & & 12 \\
\hline
\end{tabular}

uncertainty due to the shape uncertainty of the acceptance correction determined from mixed events in $\Delta \eta$ is correlated for different angles relative to the event plane and uncorrelated between different $p_{\mathrm{T}}^{\text {assoc }}$ bins. The uncertainty due to the background subtraction is nontrivially correlated for different angles of the jet relative to the event plane but uncorrelated between different $p_{\mathrm{T}}^{\text {assoc }}$ bins.

\section{F. Impact of event plane resolution}

To understand the impact of a possible event plane dependence in the signal, we consider the Fourier decomposition approach to correcting for the event plane resolution as in Ref. [72]. We can quantify the true azimuthal anisotropy of the signal by a Fourier decomposition as

$$
S(\Delta \phi)\left(1+2 \sum_{n=1}^{\infty} v_{n}^{\text {quench }} \cos \left(\phi^{\text {trig }}-\psi\right)\right),
$$

where $S(\Delta \phi)$ is the correlation function of the signal averaged over all angles relative to the event plane, the $v_{n}^{\text {quench }}$ are due to jet quenching, and $\phi^{\text {trig }}$ is the azimuthal angle of the trigger particle. The $v_{n}^{\text {quench }}$ could also be a function of $\Delta \phi$. The measured azimuthal anisotropy of the signal is then given by

$$
S\left(1+2 \sum_{n=1}^{\infty} \Re_{n} v_{n}^{\text {quench }} \cos \left(\phi^{\text {trig }}-\psi\right)\right) .
$$

Note that the $v_{n}^{\text {quench }}$ are distinct from both the $v_{n}$ from flow and the jet $v_{n}$. The jet $v_{n}$ measured in Refs. $[33,34]$ are anisotropies in the number of jets relative to the event plane while the $v_{n}^{\text {quench }}$ are a measure of the anisotropies of the constituents of those jets. Precision extraction of the $v_{n}^{\text {quench }}$ would require measurements of the signal in several bins of $\phi^{\text {trig }}-\psi$ and is not feasible for this measurement. Equation (9) shows that the impact of the finite event plane resolution is small, since for this analysis $\mathfrak{R}_{2}=0.73$.
If we assume that the $v_{n}^{\text {quench }}$ do not depend on $\Delta \phi$, the yields are given by integrating Eq. (9) over the same angles of the trigger particle relative to the event plane. The in-plane $\left(Y_{\mathrm{IP}}\right)$, mid-plane $\left(Y_{\mathrm{MP}}\right)$, and out-of-plane $\left(Y_{\mathrm{OP}}\right)$ yields up to $n=$ 3 in terms of the average yield $(Y)$ are then given by

$$
\begin{aligned}
Y_{\mathrm{IP}} & =Y\left(1+\frac{6}{\pi} \Re_{1} v_{1}^{\text {quench }}+\frac{3 \sqrt{3}}{\pi} \mathfrak{R}_{2} v_{2}^{\text {quench }}+\frac{4}{\pi} \mathfrak{R}_{3} v_{3}^{\text {quench }}\right), \\
Y_{\mathrm{MP}} & =Y\left(1+\frac{6(\sqrt{3}-1)}{\pi} \Re_{1} v_{1}^{\text {quench }}-\frac{4}{\pi} \mathfrak{R}_{3} v_{3}^{\text {quench }}\right), \\
Y_{\mathrm{OP}} & =Y\left(1-\frac{3 \sqrt{3}}{\pi} \mathfrak{R}_{2} v_{2}^{\text {quench }}\right) .
\end{aligned}
$$

The differences and ratios of the yields up to $n=3$ are then

$$
\begin{aligned}
\frac{Y_{\mathrm{OP}}}{Y_{\mathrm{IP}}} \approx & \frac{Y_{\mathrm{OP}}-Y_{\mathrm{IP}}}{Y} \\
\approx & 1-\frac{6}{\pi} \Re_{1} v_{1}^{\text {quench }}-\frac{6 \sqrt{3}}{\pi} \mathfrak{R}_{2} v_{2}^{\text {quench }}-\frac{4}{\pi} \mathfrak{R}_{3} v_{3}^{\text {quench }}, \\
\frac{Y_{\mathrm{MP}}}{Y_{\mathrm{IP}}} \approx & \frac{Y_{\mathrm{MP}}-Y_{\mathrm{IP}}}{Y} \\
\approx & 1-\frac{6 \sqrt{3}-12}{\pi} \Re_{1} v_{1}^{\text {quench }}-\frac{3 \sqrt{3}}{\pi} \mathfrak{R}_{2} v_{2}^{\text {quench }} \\
& -\frac{8}{\pi} \mathfrak{R}_{3} v_{3}^{\text {quench }} .
\end{aligned}
$$

Since the coefficients of the $v_{n}^{\text {quench }}$ are on the order of 1 , the deviations of these ratios from 1 are on the order of the $v_{n}^{\text {quench }}$. The odd $v_{n}^{\text {quench }}$ will partially cancel out because they will have opposite signs on different sides of the event plane and in the absence of surface bias, they will cancel out completely. The $n=2$ term is therefore likely the dominant term. We use this expression to evaluate the approximate effect of 


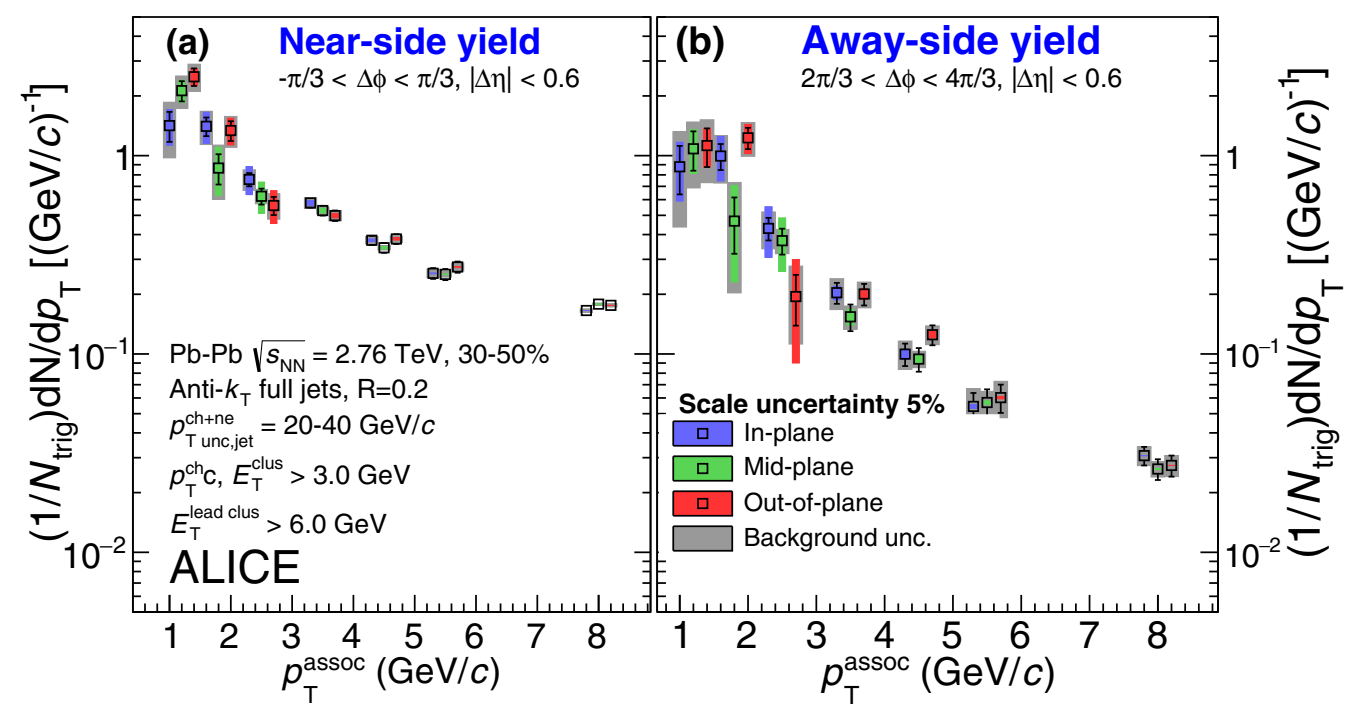

FIG. 4. The (a) near-side and (b) away-side yield vs $p_{\mathrm{T}}^{\text {assoc }}$ for $20<p_{\mathrm{T}}^{\text {jet }}<40 \mathrm{GeV} / c$ full jets of $30-50 \%$ centrality in Pb-Pb collisions. The wider band corresponds to the background uncertainty, which is nontrivially correlated point-to-point [27,37]. The narrower bands are the systematic uncertainties coming from the shape uncertainty of the acceptance correction. There is an additional 5\% global scale uncertainty. Points are displaced for visibility.

the event plane resolution. The allowed range of $v_{n}^{\text {quench }}$ is $-0.5<v_{2}^{\text {quench }}<0.5$, with positive (negative) values indicating suppression (enhancement) of the constituents. While the $v_{n}^{\text {quench }}$ is a measure of the asymmetry of modifications of jets relative to the event plane rather than the distribution of jets, we consider the asymmetry in the number of jets [33,34], $v_{2}^{\text {jet }}=0.1$, as a reasonable value of $v_{n}^{\text {quench }}$. This would lead to $\frac{Y_{\mathrm{OP}}}{Y_{\mathrm{IP}}} \approx \frac{Y_{\mathrm{OP}}-Y_{\mathrm{IP}}}{Y} \approx 0.67$ and $\frac{Y_{\mathrm{MP}}}{Y_{\mathrm{IP}}} \approx \frac{Y_{\mathrm{MP}}-Y_{\mathrm{IP}}}{Y} \approx 0.83$ with perfect event plane resolution $\left(\Re_{2}=1.0\right)$ and $\frac{Y_{\mathrm{OP}}}{Y_{\mathrm{IP}}} \approx \frac{Y_{\mathrm{OP}}-Y_{\mathrm{IP}}}{Y} \approx 0.75$ and $\frac{Y_{\mathrm{MP}}}{Y_{\mathrm{IP}}} \approx \frac{Y_{\mathrm{MP}}-Y_{\mathrm{IP}}}{Y} \approx 0.88$ with the event plane resolution in this analysis, $\mathfrak{R}_{2}=0.73$.

\section{RESULTS}

The near-side and away-side jet yields as a function of $p_{\mathrm{T}}^{\text {assoc }}$ for $20<p_{\mathrm{T}}^{\text {jet }}<40 \mathrm{GeV} / c$ full jets in 30$50 \%$ central $\mathrm{Pb}-\mathrm{Pb}$ collisions are shown in Fig. 4 for jets reconstructed in-plane, mid-plane, and out-of plane for $1.0<p_{\mathrm{T}}^{\text {assoc }}<1.5 \mathrm{GeV} / c, 1.5<p_{\mathrm{T}}^{\text {assoc }}<2.0 \mathrm{GeV} / c$, $2.0<p_{\mathrm{T}}^{\text {assoc }}<3.0 \mathrm{GeV} / c, 3.0<p_{\mathrm{T}}^{\text {assoc }}<4.0 \mathrm{GeV} / c, 4.0<$ $p_{\mathrm{T}}^{\text {assoc }}<5.0 \mathrm{GeV} / c, 5.0<p_{\mathrm{T}}^{\text {assoc }}<6.0 \mathrm{GeV} / c$, and $6.0<$ $p_{\mathrm{T}}^{\text {assoc }}<10.0 \mathrm{GeV} / c$. The dominant feature is the decrease in the yield with increasing $p_{\mathrm{T}}^{\text {assoc }}$. Note that yields with $p_{\mathrm{T}}^{\text {assoc }}>3 \mathrm{GeV} / c$ include jet constituents, complicating the interpretation of these data points. We therefore focus on lower momentum on the near side and on the away side.

Jet-hadron correlations can be used to measure changes in the momentum balance within the jet, as in Ref. [24]. Partonic energy loss will shift energy in the jet from higher momentum constituents to lower momentum constituents, so if jets in-plane interact less with the medium, the differences $Y_{\mathrm{MP}}-Y_{\mathrm{IP}}$ and $Y_{\mathrm{OP}}-Y_{\mathrm{IP}}$ will be negative at high momenta and positive at low momenta. For these differences, the systematic uncertainties partially cancel out. Figure 5 shows the yield differences $Y_{\mathrm{MP}}-Y_{\mathrm{IP}}$ and $Y_{\mathrm{OP}}-Y_{\mathrm{IP}}$ for the near and away side. There is no event plane dependence within uncertainties, consistent with expectations if $v_{2}^{\text {quench }} \approx 0.1$ as observed for inclusive jet production. Comparisons between yields in jethadron correlations in $\mathrm{Au}-\mathrm{Au}$ and $\mathrm{pp}$ collisions demonstrated suppression at high momenta and an enhancement at low momenta in Au-Au collisions at $\sqrt{s_{N N}}=200 \mathrm{GeV}$ [24]. The lack of an event plane dependence therefore indicates that any dependence of these modifications on the average path length is less than our experimental uncertainties.

To better quantify and examine the event plane dependence of the yields, ratios of mid-plane yields relative to in-plane yields $Y_{\mathrm{MP}} / Y_{\mathrm{IP}}$ and out-of-plane yields relative to in-plane yields $Y_{\mathrm{OP}} / Y_{\mathrm{IP}}$ as a function of $p_{\mathrm{T}}^{\text {assoc }}$ are shown in Fig. 6 for both the near and away sides. As for the yield differences, a substantial fraction of the systematic uncertainties cancel out for the ratios. If medium modifications increase with increasing path length traversed by the parton, these ratios will be less than 1 at high momenta and greater than 1 at low momenta. These ratios are consistent with one for all $p_{\mathrm{T}}^{\text {assoc }}$. In contrast, $R_{\mathrm{AA}}$ can be as low as 0.1 [7], indicating partonic energy loss.

The modification of the correlated yield ratios $Y_{\mathrm{OP}} / Y_{\mathrm{IP}}$ and $Y_{\mathrm{MP}} / Y_{\mathrm{IP}}$ due to jet quenching can be estimated from Eq. (11) as approximately $1-3.3 \times \mathfrak{R}_{2} v_{2}^{\text {quench }}$ for out-of-plane to inplane ratios and $1-1.7 \times \Re_{2} v_{2}^{\text {quench }}$ for mid-plane to in-plane ratios following the logic in Sec. III F. Since $\mathfrak{R}_{2}=0.73$, the ratios in Fig. 6 can be used to constrain a hypothetical $v_{2}^{\text {quench }}$. While $v_{2}^{\text {quench }}$ is a measure of the azimuthal asymmetry in jet modifications rather than the number of jets, we use the asymmetry in the number of jets, $v_{2}^{\text {jet }}=0.1[33,34]$, as a reasonable value for $v_{2}^{\text {quench }}$. If $v_{2}^{\text {quench }}=0.1$, the out-of-plane 


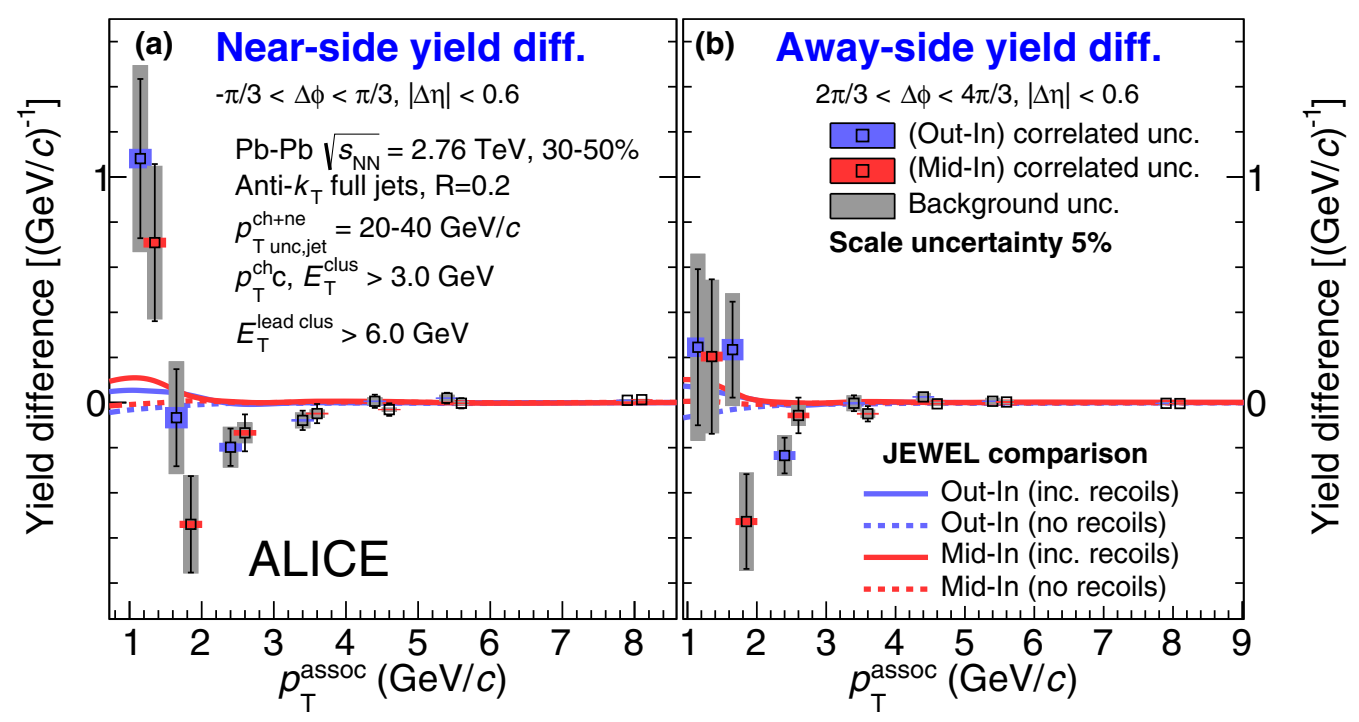

FIG. 5. The (a) near-side and (b) away-side yield differences vs $p_{\mathrm{T}}^{\text {assoc }}$ for $20<p_{\mathrm{T}}^{\text {jet }}<40 \mathrm{GeV} / c$ full jets of $30-50 \%$ centrality in $\mathrm{Pb}-\mathrm{Pb}$ collisions. The narrower band corresponds to the background uncertainty, which is nontrivially correlated point-to-point [27,37]. The wider bands are the systematic uncertainties coming from the shape uncertainty of the acceptance correction. There is an additional 5\% global scale uncertainty. Points are displaced for visibility. Data are compared to calculations from JEWEL [73] with and without recoil particles.

to in-plane ratios would be 0.75 and the mid-plane to inplane ratios would be 0.88. The data in Fig. 6 are therefore consistent both with $v_{2}^{\text {quench }}$ comparable to the inclusive jet asymmetry and with no asymmetry.

To investigate whether or not there is a systematic change in the ratio of yields with the angle of the jet relative to the event plane, we fit the data in Fig. 6 to a constant. The systematic uncertainties are treated as uncorrelated point-topoint and added in quadrature to the statistical uncertainties. The results are given in Table III and are consistent with yield ratios of 1 . We note that medium modifications could result in a $p_{\mathrm{T}}^{\text {assoc }}$ dependence and this could be exacerbated by kinematic biases on the near side because associated particles with momenta above $3 \mathrm{GeV} / c$ are included in jet reconstruction. The $\chi^{2}$ per degree of freedom may be large either because this procedure averages over different physical effects which change with momentum or because of point-to-point correlations in the uncertainties.

Figure 7 shows the widths from a fit to the Gaussian for the near and away sides. Broadening would be expected from

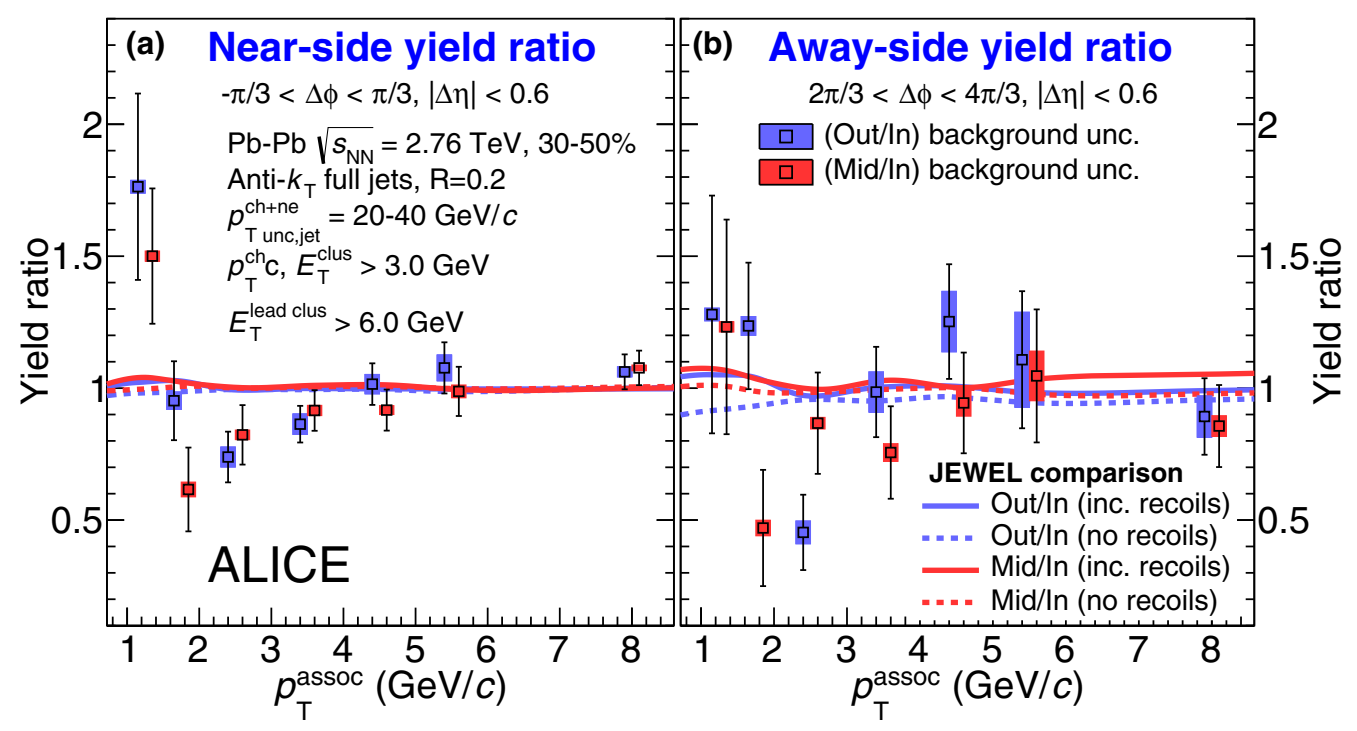

FIG. 6. The (a) near-side and (b) away-side yield ratios vs $p_{\mathrm{T}}^{\text {assoc }}$ for $20<p_{\mathrm{T}}^{\text {jet }}<40 \mathrm{GeV} / c$ full jets of $30-50 \%$ centrality in $\mathrm{Pb}-\mathrm{Pb}$ collisions. The bands correspond to the background uncertainty, which is nontrivially correlated point-to-point [27,37]. The systematic uncertainties coming from the shape uncertainty of the acceptance correction cancel out for the ratios. Points are displaced for visibility. Data are compared to calculations from JEWEL [73] with and without recoil particles. 
TABLE III. Results of fits to Fig. 6 to a constant $c$, the $\chi^{2}$ over the number of degrees of freedom (NDF), the number of standard deviations $\sigma$ of $c$ from 1 , and the range of $c$ within a $90 \%$ confidence limit (CL).

\begin{tabular}{|c|c|c|c|c|}
\hline Parameter & $Y_{\mathrm{OP}} / Y_{\mathrm{IP}}$ & $Y_{\mathrm{MP}} / Y_{\mathrm{IP}}$ & $Y_{\mathrm{OP}} / Y_{\mathrm{IP}}$ & $Y_{\mathrm{MP}} / Y_{\mathrm{IP}}$ \\
\hline$\chi^{2} / \mathrm{NDF}$ & 2.5 & 2.4 & 2.4 & 0.8 \\
\hline$\sigma$ & -0.8 & -1.1 & -1.5 & -2.1 \\
\hline $90 \% \mathrm{CL}$ & $0.91-1.03$ & $0.90-1.02$ & $0.75-1.02$ & $0.71-0.96$ \\
\hline
\end{tabular}

either collisional energy loss or gluon bremsstrahlung and path length dependent energy loss would lead to a width greater for jets out-of-plane than in-plane. No event plane dependence is observed within uncertainties, indicating that any effect is smaller than the precision of the data.

The data in Figs. 5 and 6 are compared to calculations from JEWEL, a jet energy loss model based on radiative and collisional energy loss in connection with partons sampled from a longitudinally expanding medium [73]. An important setting in the model is the choice of whether or not to keep the recoiled partons sampled from the medium in the simulation. With no recoils, the lost jet momentum vanishes from the entire system, while including the recoils conserves the jet's overall momentum, but adds energy and background particles (from the medium) to the simulated dijet. We compare to JEWEL with both recoils off and recoils on. Results with recoils off are useful for modeling energy loss in the hard part of the jet. Results with recoils on show where the jet's lost momentum goes. Any experimental analysis would likely include some but not all of the recoil particles in JEWEL, as some proportion of the recoil particles are indistinguishable from background.

JEWEL only predicts a slight event plane dependence, despite the path length dependence of partonic energy loss, due to the dominant impact of jet-by-jet fluctuations in partonic energy loss over path length dependence $[38,74]$. The slight event plane dependence predicted by JEWEL is well below the systematic uncertainty in the measurement. The agreement of JEWEL with the data is therefore consistent with path length dependence having an insignificant impact compared to jet-by-jet fluctuations in energy loss, although fluctuations in the density of the medium (not included in the JEWEL model) may also suppress observable path length dependence.

\section{CONCLUSIONS}

Partonic interactions depend on the length traversed in the medium, so any medium modifications of the jet are expected to be path length dependent. The path length traversed by a jet is correlated on average with the angle of the jet with respect to the event plane. The use of the RPF method for background subtraction reduces the assumptions required for background subtraction and since the determination of the background is currently limited by statistics, it is likely that future studies could reduce these systematic uncertainties. Measurements of jet-hadron correlations relative to the event plane in $\mathrm{Pb}-\mathrm{Pb}$ collisions at $\sqrt{s_{N N}}=2.76 \mathrm{TeV}$ are presented. Results are consistent with no dependence in the yields or the widths on the angle of the jet relative to the event plane within uncertainties. This may indicate that jet-by-jet fluctuations in

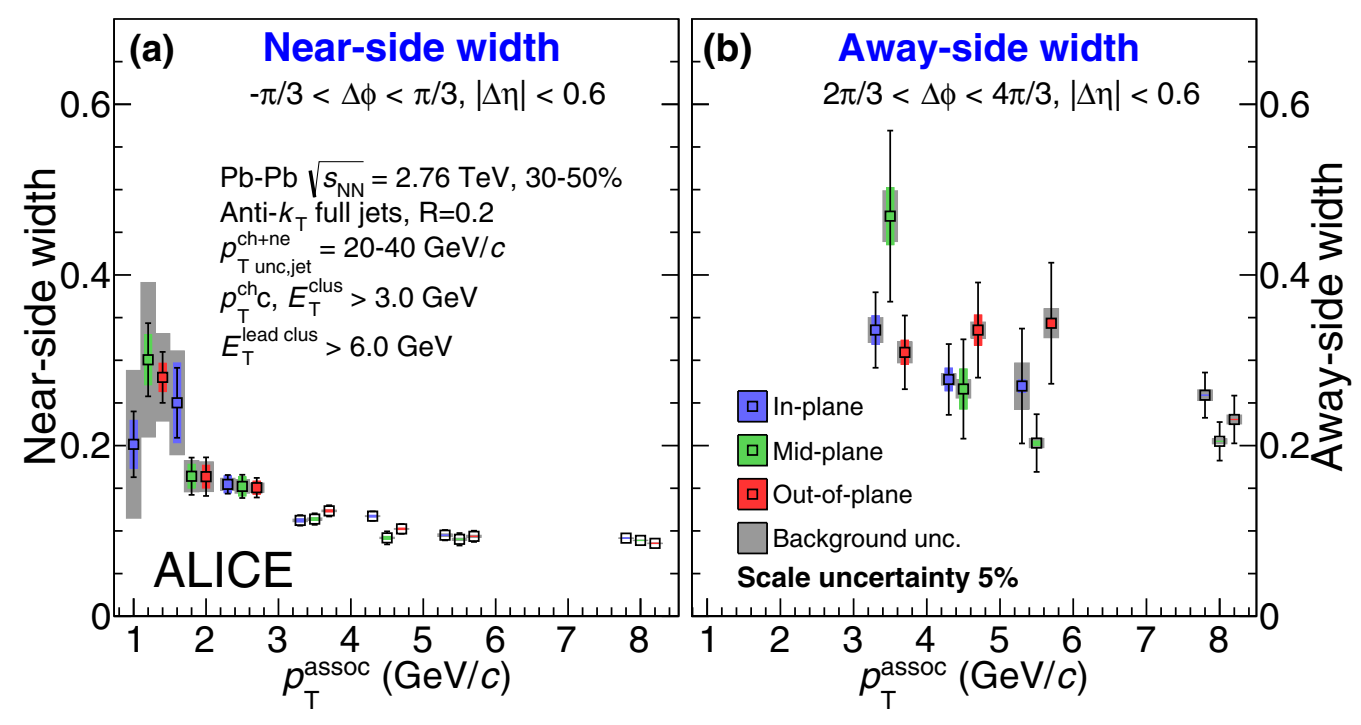

FIG. 7. The (a) near-side and (b) away-side widths vs $p_{\mathrm{T}}^{\text {assoc }}$ for $20<p_{\mathrm{T}}^{\text {jet }}<40 \mathrm{GeV} / c$ full jets of $30-50 \%$ centrality in $\mathrm{Pb}-\mathrm{Pb}$ collisions. The wider band corresponds the background uncertainty, which is nontrivially correlated point-to-point [27,37]. The narrower bands are the systematic uncertainties coming from the shape uncertainty of the acceptance correction. Points are displaced for visibility. 
partonic energy loss are important for a full description of medium modifications of jets.

\section{ACKNOWLEDGMENTS}

The ALICE Collaboration would like to thank all its engineers and technicians for their invaluable contributions to the construction of the experiment and the CERN accelerator teams for the outstanding performance of the LHC complex. The ALICE Collaboration gratefully acknowledges the resources and support provided by all Grid centers and the Worldwide LHC Computing Grid (WLCG) collaboration. The ALICE Collaboration acknowledges the following funding agencies for their support in building and running the ALICE detector: A. I. Alikhanyan National Science Laboratory (Yerevan Physics Institute) Foundation (ANSL), State Committee of Science and World Federation of Scientists (WFS), Armenia; Austrian Academy of Sciences, Austrian Science Fund (FWF): [M 2467-N36] and Nationalstiftung für Forschung, Technologie und Entwicklung, Austria; Ministry of Communications and High Technologies, National Nuclear Research Center, Azerbaijan; Conselho Nacional de Desenvolvimento Científico e Tecnológico (CNPq), Financiadora de Estudos e Projetos (Finep), Fundação de Amparo à Pesquisa do Estado de São Paulo (FAPESP) and Universidade Federal do Rio Grande do Sul (UFRGS), Brazil; Ministry of Education of China (MOEC), Ministry of Science \& Technology of China (MSTC) and National Natural Science Foundation of China (NSFC), China; Ministry of Science and Education and Croatian Science Foundation, Croatia; Centro de Aplicaciones Tecnológicas y Desarrollo Nuclear (CEADEN), Cubaenergía, Cuba; Ministry of Education, Youth and Sports of the Czech Republic, Czech Republic; The Danish Council for Independent Research-Natural Sciences, the VILLUM FONDEN and Danish National Research Foundation (DNRF), Denmark; Helsinki Institute of Physics (HIP), Finland; Commissariat à l'Energie Atomique (CEA), Institut National de Physique Nucléaire et de Physique des Particules (IN2P3) and Centre National de la Recherche Scientifique (CNRS) and Région des Pays de la Loire, France; Bundesministerium für Bildung und Forschung (BMBF) and GSI Helmholtzzentrum für Schwerionenforschung $\mathrm{GmbH}$, Germany; General Secretariat for Research and Technology, Ministry of Education, Research and Religions, Greece; National Research, Development and Innovation Office, Hungary; Department of
Atomic Energy Government of India (DAE), Department of Science and Technology, Government of India (DST), University Grants Commission, Government of India (UGC) and Council of Scientific and Industrial Research (CSIR), India; Indonesian Institute of Science, Indonesia; Centro Fermi Museo Storico della Fisica e Centro Studi e Ricerche Enrico Fermi and Istituto Nazionale di Fisica Nucleare (INFN), Italy; Institute for Innovative Science and Technology, Nagasaki Institute of Applied Science (IIST), Japanese Ministry of Education, Culture, Sports, Science and Technology (MEXT) and Japan Society for the Promotion of Science (JSPS) KAKENHI, Japan; Consejo Nacional de Ciencia (CONACYT) y Tecnología, through Fondo de Cooperación Internacional en Ciencia y Tecnología (FONCICYT) and Dirección General de Asuntos del Personal Academico (DGAPA), Mexico; Nederlandse Organisatie voor Wetenschappelijk Onderzoek (NWO), Netherlands; The Research Council of Norway, Norway; Commission on Science and Technology for Sustainable Development in the South (COMSATS), Pakistan; Pontificia Universidad Católica del Perú, Peru; Ministry of Science and Higher Education and National Science Centre, Poland; Korea Institute of Science and Technology Information and National Research Foundation of Korea (NRF), Republic of Korea; Ministry of Education and Scientific Research, Institute of Atomic Physics and Ministry of Research and Innovation and Institute of Atomic Physics, Romania; Joint Institute for Nuclear Research (JINR), Ministry of Education and Science of the Russian Federation, National Research Centre Kurchatov Institute, Russian Science Foundation and Russian Foundation for Basic Research, Russia; Ministry of Education, Science, Research and Sport of the Slovak Republic, Slovakia; National Research Foundation of South Africa, South Africa; Swedish Research Council (VR) and Knut \& Alice Wallenberg Foundation (KAW), Sweden; European Organization for Nuclear Research, Switzerland; Suranaree University of Technology (SUT), National Science and Technology Development Agency (NSDTA) and Office of the Higher Education Commission under NRU project of Thailand, Thailand; Turkish Atomic Energy Agency (TAEK), Turkey; National Academy of Sciences of Ukraine, Ukraine; Science and Technology Facilities Council (STFC), United Kingdom; National Science Foundation of the United States of America (NSF) and United States Department of Energy, Office of Nuclear Physics (DOE NP), United States of America.
[1] K. Adcox et al. (PHENIX Collaboration), Formation of dense partonic matter in relativistic nucleus-nucleus collisions at RHIC: Experimental evaluation by the PHENIX collaboration, Nucl. Phys. A 757, 184 (2005).

[2] J. Adams et al. (STAR Collaboration), Experimental and theoretical challenges in the search for the quark gluon plasma: The STAR collaboration's critical assessment of the evidence from RHIC collisions, Nucl. Phys. A 757, 102 (2005).

[3] B. B. Back et al. (PHOBOS Collaboration), The PHOBOS perspective on discoveries at RHIC, Nucl. Phys. A 757, 28 (2005).
[4] I. Arsene et al. (BRAHMS Collaboration), Quark Gluon Plasma and Color Glass Condensate at RHIC? The perspective from the BRAHMS experiment, Nucl. Phys. A 757, 1 (2005).

[5] K. Aamodt et al. (ALICE Collaboration), Two-pion BoseEinstein correlations in central $\mathrm{Pb}-\mathrm{Pb}$ collisions at $\sqrt{s_{N N}}=$ 2.76 TeV, Phys. Lett. B 696, 328 (2011).

[6] K. Aamodt et al. (ALICE Collaboration), Centrality Dependence of the Charged-Particle Multiplicity Density at MidRapidity in $\mathrm{Pb}-\mathrm{Pb}$ Collisions at $\sqrt{s_{N N}}=2.76$, Phys. Rev. Lett. 106, 032301 (2011). 
[7] K. Aamodt et al. (ALICE Collaboration), Suppression of Charged Particle Production at Large Transverse Momentum in Central $\mathrm{Pb}-\mathrm{Pb}$ Collisions at $\sqrt{s_{N N}}=2.76 \mathrm{TeV}$, Phys. Lett. B 696, 30 (2011).

[8] K. Aamodt et al. (ALICE Collaboration), Charged-Particle Multiplicity Density at Mid-Rapidity in Central Pb-Pb Collisions at $\sqrt{s_{N N}}=2.76 \mathrm{TeV}$, Phys. Rev. Lett. 105, 252301 (2010).

[9] K. Aamodt et al. (ALICE Collaboration), Elliptic Flow of Charged Particles in $\mathrm{Pb}-\mathrm{Pb}$ Collisions at 2.76 TeV, Phys. Rev. Lett. 105, 252302 (2010).

[10] G. Aad et al. (ATLAS Collaboration), Observation of a Centrality-Dependent Dijet Asymmetry in Lead-Lead Collisions at $\sqrt{s_{N N}}=2.76 \mathrm{TeV}$ with the ATLAS Detector at the LHC, Phys. Rev. Lett. 105, 252303 (2010).

[11] S. Chatrchyan et al. (CMS Collaboration), Observation and studies of jet quenching in $\mathrm{PbPb}$ collisions at nucleon-nucleon center-of-mass energy $\sqrt{s_{N N}}=2.76 \mathrm{TeV}$, Phys. Rev. C 84, 024906 (2011).

[12] M. Connors, C. Nattrass, R. Reed, and S. Salur, Jet measurements in heavy ion physics, Rev. Mod. Phys. 90, 025005 (2018).

[13] G.-Y. Qin and X.-N. Wang, Jet quenching in high-energy heavyion collisions, Int. J. Mod. Phys. E 24, 1530014 (2015)

[14] J. Adams et al. (STAR Collaboration), Transverse Momentum and Collision Energy Dependence of High $p_{T}$ Hadron Suppression in $\mathrm{Au}+\mathrm{Au}$ Collisions at Ultrarelativistic Energies, Phys. Rev. Lett. 91, 172302 (2003).

[15] S. Adler et al. (PHENIX Collaboration), Suppressed $\pi^{0}$ Production at Large Transverse Momentum in Central $\mathrm{Au}+\mathrm{Au}$ Collisions at $\sqrt{s_{N N}}=200 \mathrm{GeV}$, Phys. Rev. Lett. 91, 072301 (2003).

[16] B. Back et al. (PHOBOS Collaboration), Pseudorapidity dependence of charged hadron transverse momentum spectra in $d+$ Au collisions at $\sqrt{s_{N N}}=200 \mathrm{GeV}$, Phys. Rev. C 70, 061901 (2004).

[17] S. Chatrchyan et al. (CMS Collaboration), Study of high- $p_{T}$ charged particle suppression in $\mathrm{PbPb}$ compared to $p p$ collisions at $\sqrt{s_{N N}}=2.76 \mathrm{TeV}$, Eur. Phys. J. C 72, 1945 (2012).

[18] K. M. Burke et al. (JET Collaboration), Extracting the jet transport coefficient from jet quenching in high-energy heavyion collisions, Phys. Rev. C 90, 014909 (2014).

[19] G. Aad et al. (ATLAS Collaboration), Measurement of inclusive jet charged-particle fragmentation functions in $\mathrm{Pb}+\mathrm{Pb}$ collisions at $\sqrt{s_{N N}}=2.76 \mathrm{TeV}$ with the ATLAS detector, Phys. Lett. B 739, 320 (2014).

[20] S. Chatrchyan et al. (CMS Collaboration), Measurement of jet fragmentation in $\mathrm{PbPb}$ and pp collisions at $\sqrt{s_{N N}}=2.76 \mathrm{TeV}$, Phys. Rev. C 90, 024908 (2014).

[21] S. Chatrchyan et al. (CMS Collaboration), Measurement of jet fragmentation into charged particles in $p p$ and $\mathrm{PbPb}$ collisions at $\sqrt{s_{N N}}=2.76 \mathrm{TeV}$, J. High Energy Phys. 10 (2012) 087.

[22] A. Adare et al. (PHENIX Collaboration), Medium Modification of Jet Fragmentation in $\mathrm{Au}+\mathrm{Au}$ Collisions at $\sqrt{s_{N N}}=$ $200 \mathrm{GeV}$ Measured in Direct Photon-Hadron Correlations, Phys. Rev. Lett. 111, 032301 (2013).

[23] G. Agakishiev et al. (STAR Collaboration), System size and energy dependence of near-side di-hadron correlations, Phys. Rev. C 85, 014903 (2012).

[24] L. Adamczyk et al. (STAR Collaboration), Jet-Hadron Correlations in $\sqrt{s_{N N}}=200 \mathrm{GeV} p+p$ and Central $\mathrm{Au}+\mathrm{Au}$ Collisions, Phys. Rev. Lett. 112, 122301 (2014).
[25] V. Khachatryan et al. (CMS Collaboration), Correlations between jets and charged particles in $\mathrm{PbPb}$ and $p p$ collisions at $\sqrt{s_{N N}}=2.76 \mathrm{TeV}$, J. High Energy Phys. 02 (2016) 156.

[26] S. Acharya et al. (ALICE Collaboration), Measurement of jet radial profiles in $\mathrm{Pb}-\mathrm{Pb}$ collisions at $\sqrt{s_{N N}}=2.76 \mathrm{TeV}$, Phys. Lett. B 796, 204 (2019).

[27] N. Sharma, J. Mazer, M. Stuart, and C. Nattrass, Background subtraction methods for precision measurements of di-hadron and jet-hadron correlations in heavy ion collisions, Phys. Rev. C 93, 044915 (2016).

[28] S. Afanasiev et al. (PHENIX Collaboration), High- $p_{T} \pi^{0}$ Production with Respect to the Reaction Plane in $\mathrm{Au}+\mathrm{Au}$ Collisions at $\sqrt{s_{N N}}=200-\mathrm{GeV}$, Phys. Rev. C 80, 054907 (2009).

[29] A. Adare et al. (PHENIX Collaboration), Azimuthal anisotropy of $\pi^{0}$ and $\eta$ mesons in $\mathrm{Au}+\mathrm{Au}$ collisions at $\sqrt{s_{N N}}=200 \mathrm{GeV}$, Phys. Rev. C 88, 064910 (2013).

[30] B. Abelev et al. (ALICE Collaboration), Anisotropic flow of charged hadrons, pions and (anti-)protons measured at high transverse momentum in $\mathrm{Pb}-\mathrm{Pb}$ collisions at $\sqrt{s_{N N}}=2.76 \mathrm{TeV}$, Phys. Lett. B 719, 18 (2013)

[31] S. Chatrchyan et al. (CMS Collaboration), Azimuthal Anisotropy of Charged Particles at High Transverse Momenta in $\mathrm{Pb}-\mathrm{Pb}$ Collisions at $\sqrt{s_{N N}}=2.76 \mathrm{TeV}$, Phys. Rev. Lett. 109, 022301 (2012).

[32] S. Acharya et al. (ALICE Collaboration), Anisotropic flow of identified particles in $\mathrm{Pb}-\mathrm{Pb}$ collisions at $\sqrt{s_{N N}}=5.02 \mathrm{TeV}$, J. High Energy Phys. 09 (2018) 006.

[33] J. Adam et al. (ALICE Collaboration), Azimuthal anisotropy of charged jet production in $\sqrt{s_{N N}}=2.76 \mathrm{TeV} \mathrm{Pb}-\mathrm{Pb}$ collisions, Phys. Lett. B 753, 511 (2016).

[34] G. Aad et al. (ATLAS Collaboration), Measurement of the Azimuthal Angle Dependence of Inclusive Jet Yields in $\mathrm{Pb}+\mathrm{Pb}$ Collisions at $\sqrt{s_{N N}}=2.76 \mathrm{TeV}$ with the ATLAS detector, Phys. Rev. Lett. 111, 152301 (2013).

[35] A. Adare et al. (PHENIX Collaboration), Suppression of away-side jet fragments with respect to the reaction plane in $\mathrm{Au}+\mathrm{Au}$ collisions at $\sqrt{s_{N N}}=200 \mathrm{GeV}$, Phys. Rev. C 84, 024904 (2011).

[36] H. Agakishiev et al. (STAR Collaboration), Event-planedependent dihadron correlations with harmonic $v_{n}$ subtraction in $\mathrm{Au}+\mathrm{Au}$ collisions at $\sqrt{s_{N N}}=200 \mathrm{GeV}$, Phys. Rev. C 89, 041901 (2014).

[37] C. Nattrass, N. Sharma, J. Mazer, M. Stuart, and A. Bejnood, Disappearance of the Mach Cone in heavy ion collisions, Phys. Rev. C 94, 011901 (2016).

[38] K. C. Zapp, Geometrical aspects of jet quenching in JEWEL, Phys. Lett. B 735, 157 (2014).

[39] J. Noronha-Hostler, B. Betz, J. Noronha, and M. Gyulassy, Event-by-Event Hydrodynamics + Jet Energy Loss: A Solution to the $R_{A A} \otimes v_{2}$ puzzle, Phys. Rev. Lett. 116, 252301 (2016).

[40] B. Betz, M. Gyulassy, M. Luzum, J. Noronha, J. NoronhaHostler, I. Portillo, and C. Ratti, Cumulants and nonlinear response of high $p_{T}$ harmonic flow at $\sqrt{s_{N N}}=5.02 \mathrm{TeV}$, Phys. Rev. C 95, 044901 (2017).

[41] B. Abelev et al. (ALICE Collaboration), Performance of the ALICE Experiment at the CERN LHC, Int. J. Mod. Phys. A 29, 1430044 (2014).

[42] E. Abbas et al. (ALICE Collaboration), Performance of the ALICE VZERO system, JINST 8, P10016 (2013). 
[43] P. Cortese et al. (ALICE Collaboration), ALICE technical design report on forward detectors: FMD, T0, and V0, CERNLHCC-2004-025 (2004), https://cds.cern.ch/record/781854.

[44] K. Aamodt et al. (ALICE Collaboration), Alignment of the ALICE Inner Tracking System with cosmic-ray tracks, J. Instrum. 5, P03003 (2010).

[45] J. Alme et al., The ALICE TPC, a large 3-dimensional tracking device with fast readout for ultra-high multiplicity events, Nucl. Instrum. Methods Phys. Res. A 622, 316 (2010).

[46] U. Abeysekara et al. (ALICE EMCal Collaboration), ALICE EMCal Physics Performance Report, arXiv:1008.0413.

[47] J. Adam et al. (ALICE Collaboration), Measurement of jet suppression in central $\mathrm{Pb}-\mathrm{Pb}$ collisions at $\sqrt{s_{N N}}=2.76 \mathrm{TeV}$, Phys. Lett. B 746, 1 (2015).

[48] G. Dellacasa et al. (ALICE Collaboration), ALICE technical design report of the inner tracking system (ITS), CERN-LHCC99-12, 1999, https://cds.cern.ch/record/401974?ln=en.

[49] G. Dellacasa et al. (ALICE Collaboration), ALICE: Technical design report of the time projection chamber, CERN-OPEN2000-183, CERN-LHCC-2000-001, 2000, https://cds.cern.ch/ record/451098.

[50] X.-N. Wang and M. Gyulassy, HIJING: A Monte Carlo model for multiple jet production in pp, pA, and AA collisions, Phys. Rev. D 44, 3501 (1991).

[51] R. Brun, F. Bruyant, F. Carminati, S. Giani, M. Maire, A. McPherson, G. Patrick, and L. Urban, GEANT Detector Description and Simulation Tool, CERN-W5013, CERN-W-5013, W5013, W-5013, 1994, https://cds.cern.ch/record/1082634.

[52] J. Adam et al. (ALICE Collaboration), Jet-like correlations with neutral pion triggers in $p p$ and central $\mathrm{Pb}-\mathrm{Pb}$ collisions at 2.76 TeV, Phys. Lett. B 763, 238 (2016).

[53] B. Abelev et al. (ALICE Collaboration), Measurement of the inclusive differential jet cross section in $p p$ collisions at $\sqrt{s}=$ 2.76 TeV, Phys. Lett. B 722, 262 (2013).

[54] B. Abelev et al. (ALICE Collaboration), Measurement of charged jet suppression in $\mathrm{Pb}-\mathrm{Pb}$ collisions at $\sqrt{s_{N N}}=$ 2.76 TeV, J. High Energy Phys. 03 (2014) 013.

[55] J. Allen et al. (ALICE EMCal Collaboration), Performance of prototypes for the ALICE electromagnetic calorimeter, Nucl. Instrum. Methods Phys. Res. A 615, 6 (2010).

[56] P. Cortese et al. (ALICE Collaboration), ALICE electromagnetic calorimeter Technical design report, CERN-LHCC2008-014, CERN-ALICE-TDR-014, 2008, https://cds.cern.ch/ record $/ 1121574 ? \ln =\mathrm{en}$.

[57] M. Cacciari, G. P. Salam, and G. Soyez, FastJet User Manual, Eur. Phys. J. C 72, 1896 (2012).

[58] B. Abelev et al. (ALICE Collaboration), Measurement of Event Background Fluctuations for Charged Particle Jet Reconstruction in $\mathrm{Pb}-\mathrm{Pb}$ collisions at $\sqrt{s_{N N}}=2.76 \mathrm{TeV}$, J. High Energy Phys. 03 (2012) 053.
[59] T. Sjostrand, S. Mrenna, and P. Z. Skands, PYTHIA 6.4 Physics and Manual, J. High Energy Phys. 05 (2006) 026.

[60] R. Field and R. C. Group (CDF Collaboration), PYTHIA tune A, HERWIG, and JIMMY in Run 2 at CDF, Reprot No. CDFANAL-CDF-PUBLIC-7822, arXiv:hep-ph/0510198.

[61] B. Abelev et al. (ALICE Collaboration), Centrality determination of $\mathrm{Pb}-\mathrm{Pb}$ collisions at $\sqrt{s_{N N}}=2.76 \mathrm{TeV}$ with ALICE, Phys. Rev. C 88, 044909 (2013).

[62] P. Sorensen, Implications of space-momentum correlations and geometric fluctuations in heavy-ion collisions, J. Phys. G 37, 094011 (2010).

[63] B. Alver and G. Roland, Collision geometry fluctuations and triangular flow in heavy-ion collisions, Phys. Rev. C 81, 054905 (2010); 82, 039903(E) (2010).

[64] A. M. Poskanzer and S. A. Voloshin, Methods for analyzing anisotropic flow in relativistic nuclear collisions, Phys. Rev. C 58, 1671 (1998).

[65] J. Barrette et al. (E877 Collaboration), Energy and charged particle flow in a $10.8 \mathrm{~A} \mathrm{GeV} / \mathrm{c} \mathrm{Au}+\mathrm{Au}$ collisions, Phys. Rev. C 55, 1420 (1997).

[66] J. Bielcikova, S. Esumi, K. Filimonov, S. Voloshin, and J. P. Wurm, Elliptic flow contribution to two particle correlations at different orientations to the reaction plane, Phys. Rev. C 69, 021901(R) (2004).

[67] C. Nattrass and T. Todoroki, Event plane dependence of the flow modulated background in dihadron and jet-hadron correlations in heavy ion collisions, Phys. Rev. C 97, 054911 (2018).

[68] G. Aad et al. (ATLAS Collaboration), Measurement of event-plane correlations in $\sqrt{s_{N N}}=2.76 \mathrm{TeV}$ lead-lead collisions with the ATLAS detector, Phys. Rev. C 90, 024905 (2014)

[69] M. Luzum and J.-Y. Ollitrault, Directed Flow at Midrapidity in Heavy-Ion Collisions, Phys. Rev. Lett. 106, 102301 (2011).

[70] G. Aad et al. (ATLAS Collaboration), Measurement of the azimuthal anisotropy for charged particle production in $\sqrt{s_{N N}}=$ 2.76 TeV lead-lead collisions with the ATLAS detector, Phys. Rev. C 86, 014907 (2012).

[71] ALICE Collaboration, Second order event plane dependence of jet-hadron correlations in $\mathrm{Pb}-\mathrm{Pb}$ collisions at $\sqrt{s_{N N}}=2.76 \mathrm{TeV}, \quad$ https://cds.cern.ch/record/2696428. ALICE-PUBLIC-2019-004.

[72] A. Adare et al. (PHENIX Collaboration), Measurement of twoparticle correlations with respect to second- and third-order event planes in $\mathrm{Au}+\mathrm{Au}$ collisions at $\sqrt{s_{N N}}=200 \mathrm{GeV}$, Phys. Rev. C 99, 054903 (2019).

[73] K. C. Zapp, JEWEL 2.0.0: directions for use, Eur. Phys. J. C 74, 2762 (2014).

[74] J. G. Milhano and K. C. Zapp, Origins of the di-jet asymmetry in heavy ion collisions, Eur. Phys. J. C 76, 288 (2016).

S. Acharya, ${ }^{141}$ D. Adamová, ${ }^{93}$ A. Adler,${ }^{73}$ J. Adolfsson, ${ }^{79}$ M. M. Aggarwal,,${ }^{98}$ G. Aglieri Rinella,${ }^{34}$ M. Agnello, ${ }^{31}$ N. Agrawal, ${ }^{10,53}$ Z. Ahammed, ${ }^{141}$ S. Ahmad, ${ }^{17}$ S. U. Ahn, ${ }^{75}$ A. Akindinov, ${ }^{90}$ M. Al-Turany, ${ }^{105}$ S. N. Alam, ${ }^{141}$ D. S. D. Albuquerque, ${ }^{122}$ D. Aleksandrov, ${ }^{86}$ B. Alessandro, ${ }^{58}$ H. M. Alfanda, ${ }^{6}$ R. Alfaro Molina, ${ }^{71}$ B. Ali, ${ }^{17}$ Y. Ali, ${ }^{15}$ A. Alici, ${ }^{10,27,53}$ A. Alkin, ${ }^{2}$ J. Alme, ${ }^{22}$ T. Alt ${ }^{68}$ L. Altenkamper,${ }^{22}$ I. Altsybeev, ${ }^{112}$ M. N. Anaam, ${ }^{6}$ C. Andrei, ${ }^{47}$ D. Andreou, ${ }^{34}$ H. A. Andrews, ${ }^{109}$ A. Andronic, ${ }^{144}$ M. Angeletti, ${ }^{34}$ V. Anguelov, ${ }^{102}$ C. Anson, ${ }^{16}$ T. Antičić, ${ }^{106}$ F. Antinori, ${ }^{56}$ P. Antonioli, ${ }^{53}$ R. Anwar, ${ }^{125}$ N. Apadula, ${ }^{78}$ L. Aphecetche, ${ }^{114}$ H. Appelshäuser ${ }^{68}$ S. Arcelli, ${ }^{27}$ R. Arnaldi, ${ }^{58}$ M. Arratia,${ }^{78}$ I. C. Arsene, ${ }^{21}$ M. Arslandok, ${ }^{102}$ A. Augustinus, ${ }^{34}$ R. Averbeck, ${ }^{105}$ S. Aziz, ${ }^{61}$ M. D. Azmi, ${ }^{17}$ A. Badalà, ${ }^{55}$ Y. W. Baek, ${ }^{40}$ S. Bagnasco, ${ }^{58}$ 
X. Bai, ${ }^{105}$ R. Bailhache, ${ }^{68}$ R. Bala, ${ }^{99}$ A. Baldisseri, ${ }^{137}$ M. Ball, ${ }^{42}$ S. Balouza, ${ }^{103}$ R. Barbera, ${ }^{28}$ L. Barioglio, ${ }^{26}$ G. G. Barnaföldi, ${ }^{145}$ L. S. Barnby, ${ }^{92}$ V. Barret, ${ }^{134}$ P. Bartalini, ${ }^{6}$ K. Barth, ${ }^{34}$ E. Bartsch, ${ }^{68}$ F. Baruffaldi, ${ }^{29}$ N. Bastid, ${ }^{134}$ S. Basu, ${ }^{143}$ G. Batigne, ${ }^{114}$ B. Batyunya, ${ }^{74}$ D. Bauri, ${ }^{48}$ J. L. Bazo Alba, ${ }^{110}$ I. G. Bearden, ${ }^{87}$ C. Bedda, ${ }^{63}$ N. K. Behera, ${ }^{60}$ I. Belikov, ${ }^{136}$ A. D.C. Bell Hechavarria, ${ }^{144}$ F. Bellini, ${ }^{34}$ R. Bellwied, ${ }^{125}$ V. Belyaev, ${ }^{91}$ G. Bencedi, ${ }^{145}$ S. Beole, ${ }^{26}$ A. Bercuci, ${ }^{47}$ Y. Berdnikov, ${ }^{96}$ D. Berenyi, ${ }^{145}$ R. A. Bertens, ${ }^{130}$ D. Berzano, ${ }^{58}$ M. G. Besoiu, ${ }^{67}$ L. Betev, ${ }^{34}$ A. Bhasin, ${ }^{99}$ I. R. Bhat, ${ }^{99}$ M. A. Bhat, ${ }^{3}$ H. Bhatt, ${ }^{48}$ B. Bhattacharjee, ${ }^{41}$ A. Bianchi, ${ }^{26}$ L. Bianchi,${ }^{26}$ N. Bianchi, ${ }^{51}$ J. Bielčík, ${ }^{37}$ J. Bielčíková, ${ }^{93}$ A. Bilandzic,${ }^{103,117}$ G. Biro, ${ }^{145}$ R. Biswas ${ }^{3}$ S. Biswas, ${ }^{3}$ J. T. Blair, ${ }^{119}$ D. Blau, ${ }^{86}$ C. Blume,${ }^{68}$ G. Boca, ${ }^{139}$ F. Bock, ${ }^{34,94}$ A. Bogdanov, ${ }^{91}$ L. Boldizsár, ${ }^{145}$ A. Bolozdynya, ${ }^{91}$ M. Bombara, ${ }^{38}$ G. Bonomi, ${ }^{140}$ H. Borel, ${ }^{137}$ A. Borissov, ${ }^{91,144} \mathrm{H}$. Bossi, ${ }^{146}$ E. Botta, ${ }^{26}$ L. Bratrud, ${ }^{68}$ P. Braun-Munzinger, ${ }^{105}$ M. Bregant, ${ }^{121}$ T. A. Broker, ${ }^{68}$ M. Broz, ${ }^{37}$ E. J. Brucken, ${ }^{43}$ E. Bruna, ${ }^{58}$ G. E. Bruno, ${ }^{104}$ M. D. Buckland, ${ }^{127}$ D. Budnikov, ${ }^{107}$ H. Buesching, ${ }^{68}$ S. Bufalino, ${ }^{31}$ O. Bugnon, ${ }^{114}$ P. Buhler, ${ }^{113}$ P. Buncic, ${ }^{34}$ Z. Buthelezi, ${ }^{72,131}$ J. B. Butt, ${ }^{15}$ J. T. Buxton, ${ }^{95}$ S. A. Bysiak, ${ }^{118}$ D. Caffarri, ${ }^{88}$ A. Caliva, ${ }^{105}$ E. Calvo Villar, ${ }^{110}$ R. S. Camacho, ${ }^{44}$ P. Camerini, ${ }^{25}$ A. A. Capon, ${ }^{113}$ F. Carnesecchi, ${ }^{10,27}$ R. Caron, ${ }^{137}$ J. Castillo Castellanos, ${ }^{137}$ A. J. Castro, ${ }^{130}$ E. A. R. Casula, ${ }^{54}$ F. Catalano, ${ }^{31}$ C. Ceballos Sanchez, ${ }^{52}$ P. Chakraborty, ${ }^{48}$ S. Chandra, ${ }^{141}$ W. Chang, ${ }^{6}$ S. Chapeland, ${ }^{34}$ M. Chartier, ${ }^{127}$

S. Chattopadhyay, ${ }^{141}$ S. Chattopadhyay, ${ }^{108}$ A. Chauvin, ${ }^{24}$ C. Cheshkov, ${ }^{135}$ B. Cheynis, ${ }^{135}$ V. Chibante Barroso, ${ }^{34}$

D. D. Chinellato, ${ }^{122}$ S. Cho, ${ }^{60}$ P. Chochula, ${ }^{34}$ T. Chowdhury, ${ }^{134}$ P. Christakoglou, ${ }^{88}$ C. H. Christensen, ${ }^{87}$ P. Christiansen, ${ }^{79}$

T. Chujo, ${ }^{133}$ C. Cicalo, ${ }^{54}$ L. Cifarelli, ${ }^{10,27}$ F. Cindolo, ${ }^{53}$ J. Cleymans, ${ }^{124}$ F. Colamaria, ${ }^{52}$ D. Colella, ${ }^{52}$ A. Collu, ${ }^{78}$ M. Colocci, ${ }^{27}$ M. Concas, ${ }^{58, a}$ G. Conesa Balbastre, ${ }^{77}$ Z. Conesa del Valle, ${ }^{61}$ M. E. Connors, ${ }^{146}$ G. Contin, ${ }^{59,127}$ J. G. Contreras, ${ }^{37}$

T. M. Cormier, ${ }^{94}$ Y. Corrales Morales, ${ }^{26,58}$ P. Cortese, ${ }^{32}$ M. R. Cosentino, ${ }^{123}$ F. Costa, ${ }^{34}$ S. Costanza, ${ }^{139}$ P. Crochet, ${ }^{134}$

E. Cuautle, ${ }^{69}$ P. Cui, ${ }^{6}$ L. Cunqueiro, ${ }^{94}$ D. Dabrowski, ${ }^{142}$ T. Dahms, ${ }^{103,117}$ A. Dainese, ${ }^{56}$ F. P. A. Damas, ${ }^{14,137}$ M. C. Danisch, ${ }^{102}$

A. Danu, ${ }^{67}$ D. Das, ${ }^{108}$ I. Das, ${ }^{108}$ P. Das, ${ }^{84}$ P. Das, ${ }^{3}$ S. Das, ${ }^{3}$ A. Dash, ${ }^{84}$ S. Dash, ${ }^{48}$ A. Dashi, ${ }^{103}$ S. De, ${ }^{84}$ A. De Caro, ${ }^{30}$ G. de Cataldo,${ }^{52}$ C. de Conti, ${ }^{121}$ J. de Cuveland, ${ }^{39}$ A. De Falco, ${ }^{24}$ D. De Gruttola, ${ }^{10}$ N. De Marco,${ }^{58}$ S. De Pasquale, ${ }^{30} \mathrm{~S}$. Deb,${ }^{49}$

B. Debjani, ${ }^{3}$ H. F. Degenhardt, ${ }^{121}$ K. R. Deja, ${ }^{142}$ A. Deloff, ${ }^{83}$ S. Delsanto, ${ }^{26,131}$ D. Devetak, ${ }^{105}$ P. Dhankher, ${ }^{48}$ D. Di Bari, ${ }^{33}$

A. Di Mauro, ${ }^{34}$ R. A. Diaz, ${ }^{8}$ T. Dietel, ${ }^{124}$ P. Dillenseger, ${ }^{68}$ Y. Ding, ${ }^{6}$ R. Divià, ${ }^{34} \varnothing$. Djuvsland, ${ }^{22}$ U. Dmitrieva, ${ }^{62}$ A. Dobrin, ${ }^{34,67}$

B. Dönigus ${ }^{68}$ O. Dordic,${ }^{21}$ A. K. Dubey, ${ }^{141}$ A. Dubla, ${ }^{105}$ S. Dudi, ${ }^{98}$ M. Dukhishyam, ${ }^{84}$ P. Dupieux, ${ }^{134}$ R. J. Ehlers, ${ }^{146}$

V. N. Eikeland, ${ }^{22}$ D. Elia, ${ }^{52}$ H. Engel, ${ }^{73}$ E. Epple, ${ }^{146}$ B. Erazmus, ${ }^{114}$ F. Erhardt, ${ }^{97}$ A. Erokhin, ${ }^{112}$ M. R. Ersdal, ${ }^{22}$ B. Espagnon, ${ }^{61}$

G. Eulisse, ${ }^{34}$ D. Evans, ${ }^{109}$ S. Evdokimov, ${ }^{89}$ L. Fabbietti, ${ }^{103,117}$ M. Faggin, ${ }^{29}$ J. Faivre, ${ }^{77}$ F. Fan, ${ }^{6}$ A. Fantoni, ${ }^{51}$ M. Fasel, ${ }^{94}$

P. Fecchio, ${ }^{31}$ A. Feliciello, ${ }^{58}$ G. Feofilov, ${ }^{12}$ A. Fernández Téllez, ${ }^{44}$ A. Ferrero, ${ }^{137}$ A. Ferretti, ${ }^{26}$ A. Festanti, ${ }^{34}$

V. J. G. Feuillard, ${ }^{102}$ J. Figiel, ${ }^{118}$ S. Filchagin, ${ }^{107}$ D. Finogeev, ${ }^{62}$ F. M. Fionda, ${ }^{22}$ G. Fiorenza, ${ }^{52}$ F. Flor, ${ }^{125}$ S. Foertsch, ${ }^{72}$

P. Foka, ${ }^{105}$ S. Fokin, ${ }^{86}$ E. Fragiacomo, ${ }^{59}$ U. Frankenfeld, ${ }^{105}$ U. Fuchs, ${ }^{34}$ C. Furget, ${ }^{77}$ A. Furs, ${ }^{62}$ M. Fusco Girard, ${ }^{30}$

J. J. Gaardhøje, ${ }^{87}$ M. Gagliardi, ${ }^{26}$ A. M. Gago, ${ }^{110}$ A. Gal, ${ }^{136}$ C. D. Galvan, ${ }^{120}$ P. Ganoti, ${ }^{82}$ C. Garabatos, ${ }^{105}$ E. Garcia-Solis, ${ }^{11}$ K. Garg, ${ }^{28}$ C. Gargiulo, ${ }^{34}$ A. Garibli, ${ }^{85}$ K. Garner, ${ }^{144}$ P. Gasik, ${ }^{103,117}$ E. F. Gauger, ${ }^{119}$ M. B. Gay Ducati, ${ }^{70}$ M. Germain, ${ }^{114}$

J. Ghosh, ${ }^{108}$ P. Ghosh, ${ }^{141}$ S. K. Ghosh, ${ }^{3}$ P. Gianotti, ${ }^{51}$ P. Giubellino, ${ }^{58,105}$ P. Giubilato, ${ }^{29}$ P. Glässel, ${ }^{102}$ D. M. Goméz Coral, ${ }^{71}$

A. Gomez Ramirez, ${ }^{73}$ V. Gonzalez, ${ }^{105}$ P. González-Zamora, ${ }^{44}$ S. Gorbunov, ${ }^{39}$ L. Görlich, ${ }^{118}$ S. Gotovac, ${ }^{35}$ V. Grabski, ${ }^{71}$

L. K. Graczykowski, ${ }^{142}$ K. L. Graham, ${ }^{109}$ L. Greiner, ${ }^{78}$ A. Grelli, ${ }^{63}$ C. Grigoras, ${ }^{34}$ V. Grigoriev, ${ }^{91}$ A. Grigoryan, ${ }^{1}$

S. Grigoryan, ${ }^{74}$ O. S. Groettvik, ${ }^{22}$ F. Grosa,${ }^{31}$ J. F. Grosse-Oetringhaus, ${ }^{34}$ R. Grosso, ${ }^{105}$ R. Guernane, ${ }^{77} \mathrm{M}$. Guittiere, ${ }^{114}$

K. Gulbrandsen, ${ }^{87}$ T. Gunji, ${ }^{132}$ A. Gupta, ${ }^{99}$ R. Gupta, ${ }^{99}$ I. B. Guzman, ${ }^{44}$ R. Haake, ${ }^{146}$ M. K. Habib, ${ }^{105}$ C. Hadjidakis, ${ }^{61}$

H. Hamagaki, ${ }^{80}$ G. Hamar, ${ }^{145}$ M. Hamid, ${ }^{6}$ R. Hannigan, ${ }^{19}$ M. R. Haque, ${ }^{63,84}$ A. Harlenderova, ${ }^{105}$ J. W. Harris, ${ }^{146}$ A. Harton, ${ }^{11}$

J. A. Hasenbichler, ${ }^{34}$ D. Hatzifotiadou, ${ }^{10,53}$ P. Hauer, ${ }^{42}$ S. Hayashi, ${ }^{132}$ S. T. Heckel, ${ }^{68,103}$ E. Hellbär, ${ }^{68}$ H. Helstrup, ${ }^{36}$

A. Herghelegiu, ${ }^{47}$ E. G. Hernandez, ${ }^{44}$ G. Herrera Corral, ${ }^{9}$ F. Herrmann, ${ }^{144}$ K. F. Hetland, ${ }^{36}$ T. E. Hilden, ${ }^{43}$ H. Hillemanns, ${ }^{34}$

C. Hills, ${ }^{127}$ B. Hippolyte, ${ }^{136}$ B. Hohlweger, ${ }^{103}$ D. Horak, ${ }^{37}$ A. Hornung, ${ }^{68}$ S. Hornung, ${ }^{105}$ R. Hosokawa, ${ }^{16,133}$ P. Hristov, ${ }^{34}$

C. Huang, ${ }^{61}$ C. Hughes, ${ }^{130}$ P. Huhn, ${ }^{68}$ T. J. Humanic, ${ }^{95}$ H. Hushnud, ${ }^{108}$ L. A. Husova, ${ }^{144}$ N. Hussain, ${ }^{41}$ S. A. Hussain, ${ }^{15}$

D. Hutter, ${ }^{39}$ J. P. Iddon, ${ }^{34,127}$ R. Ilkaev, ${ }^{107}$ M. Inaba, ${ }^{133}$ G. M. Innocenti, ${ }^{34}$ M. Ippolitov, ${ }^{86}$ A. Isakov, ${ }^{93}$ M. S. Islam, ${ }^{108}$

M. Ivanov, ${ }^{105}$ V. Ivanov, ${ }^{96}$ V. Izucheev, ${ }^{89}$ B. Jacak, ${ }^{78}$ N. Jacazio, ${ }^{27,53}$ P. M. Jacobs, ${ }^{78}$ M. B. Jadhav, ${ }^{48}$ S. Jadlovska, ${ }^{116}$

J. Jadlovsky, ${ }^{116}$ S. Jaelani, ${ }^{63}$ C. Jahnke, ${ }^{121}$ M. J. Jakubowska, ${ }^{142}$ M. A. Janik, ${ }^{142}$ T. Janson, ${ }^{73}$ M. Jercic, ${ }^{97}$ O. Jevons, ${ }^{109}$

M. Jin, ${ }^{125}$ F. Jonas, ${ }^{94,144}$ P. G. Jones, ${ }^{109}$ J. Jung, ${ }^{68}$ M. Jung, ${ }^{68}$ A. Jusko, ${ }^{109}$ P. Kalinak, ${ }^{64}$ A. Kalweit, ${ }^{34}$ V. Kaplin, ${ }^{91}$ S. Kar, ${ }^{6}$

A. Karasu Uysal, ${ }^{76}$ O. Karavichev, ${ }^{62}$ T. Karavicheva, ${ }^{62}$ P. Karczmarczyk, ${ }^{34}$ E. Karpechev, ${ }^{62}$ U. Kebschull, ${ }^{73}$ R. Keidel ${ }^{46}$

M. Keil, ${ }^{34}$ B. Ketzer, ${ }^{42}$ Z. Khabanova, ${ }^{88}$ A. M. Khan, ${ }^{6}$ S. Khan, ${ }^{17}$ S. A. Khan, ${ }^{141}$ A. Khanzadeev, ${ }^{96}$ Y. Kharlov, ${ }^{89}$ A. Khatun, ${ }^{17}$

A. Khuntia, ${ }^{118}$ B. Kileng, ${ }^{36}$ B. Kim, ${ }^{60}$ B. Kim, ${ }^{133}$ D. Kim, ${ }^{147}$ D. J. Kim, ${ }^{126}$ E. J. Kim, ${ }^{13}$ H. Kim, ${ }^{18,147}$ J. Kim, ${ }^{147}$ J. S. Kim, ${ }^{40}$

J. Kim, ${ }^{102}$ J. Kim,${ }^{147}$ J. Kim, ${ }^{13}$ M. Kim, ${ }^{12}$ S. Kim, ${ }^{19}$ T. Kim,${ }^{147}$ T. Kim, ${ }^{147}$ S. Kirsch, ${ }^{39,68}$ I. Kisel, ${ }^{39}$ S. Kiselev, ${ }^{90}$ A. Kisiel, ${ }^{142}$

J. L. Klay, ${ }^{5}$ C. Klein, ${ }^{68}$ J. Klein, ${ }^{58}$ S. Klein, ${ }^{78}$ C. Klein-Bösing, ${ }^{144}$ M. Kleiner, ${ }^{68}$ S. Klewin, ${ }^{102}$ A. Kluge, ${ }^{34}$ M. L. Knichel, ${ }^{34}$

A. G. Knospe, ${ }^{125}$ C. Kobdaj, ${ }^{115}$ M. K. Köhler, ${ }^{102}$ T. Kollegger, ${ }^{105}$ A. Kondratyev, ${ }^{74}$ N. Kondratyeva, ${ }^{91}$ E. Kondratyuk, ${ }^{89}$

J. Konig, ${ }^{68}$ P. J. Konopka, ${ }^{34}$ L. Koska, ${ }^{116}$ O. Kovalenko, ${ }^{83}$ V. Kovalenko, ${ }^{112}$ M. Kowalski, ${ }^{118}$ I. Králik, ${ }^{64}$ A. Kravčáková, ${ }^{38}$

L. Kreis, ${ }^{105}$ M. Krivda, ${ }^{64,109}$ F. Krizek, ${ }^{93}$ K. Krizkova Gajdosova, ${ }^{37}$ M. Krüger, ${ }^{68}$ E. Kryshen, ${ }^{96}$ M. Krzewicki, ${ }^{39}$

A. M. Kubera, ${ }^{95}$ V. Kučera, ${ }^{60}$ C. Kuhn, ${ }^{136}$ P. G. Kuijer, ${ }^{88}$ L. Kumar, ${ }^{98}$ S. Kumar, ${ }^{48}$ S. Kundu ${ }^{84}$ P. Kurashvili, ${ }^{83}$ A. Kurepin, ${ }^{62}$

A. B. Kurepin, ${ }^{62}$ A. Kuryakin, ${ }^{107}$ S. Kushpil, ${ }^{93}$ J. Kvapil, ${ }^{109}$ M. J. Kweon, ${ }^{60}$ J. Y. Kwon, ${ }^{60}$ Y. Kwon, ${ }^{147}$ S. L. La Pointe, ${ }^{39}$

P. La Rocca, ${ }^{28}$ Y. S. Lai, ${ }^{78}$ R. Langoy, ${ }^{129}$ K. Lapidus, ${ }^{44}$ A. Lardeux,${ }^{21}$ P. Larionov, ${ }^{51}$ E. Laudi, ${ }^{34}$ R. Lavicka,${ }^{37}$ T. Lazareva, ${ }^{112}$

R. Lea, ${ }^{25}$ L. Leardini, ${ }^{102}$ J. Lee, ${ }^{133}$ S. Lee, ${ }^{147}$ F. Lehas, ${ }^{88}$ S. Lehner, ${ }^{113}$ J. Lehrbach, ${ }^{39}$ R. C. Lemmon, ${ }^{92}$ I. León Monzón, ${ }^{120}$

E. D. Lesser, ${ }^{20}$ M. Lettrich, ${ }^{34}$ P. Lévai, ${ }^{145}$ X. Li,${ }^{12}$ X. L. Li, ${ }^{6}$ J. Lien, ${ }^{129}$ R. Lietava, ${ }^{109}$ B. Lim, ${ }^{18}$ V. Lindenstruth, ${ }^{39}$ 
S. W. Lindsay, ${ }^{127}$ C. Lippmann, ${ }^{105}$ M. A. Lisa, ${ }^{95}$ V. Litichevskyi, ${ }^{43}$ A. Liu, ${ }^{78}$ S. Liu, ${ }^{95}$ W. J. Llope,${ }^{143}$ I. M. Lofnes, ${ }^{22}$ V. Loginov, ${ }^{91}$ C. Loizides, ${ }^{94}$ P. Loncar, ${ }^{35}$ X. Lopez, ${ }^{134}$ E. López Torres, ${ }^{8}$ J. R. Luhder, ${ }^{144}$ M. Lunardon, ${ }^{29}$ G. Luparello, ${ }^{59}$ Y. Ma, ${ }^{111}$ A. Maevskaya, ${ }^{62}$ M. Mager, ${ }^{34}$ S. M. Mahmood, ${ }^{21}$ T. Mahmoud, ${ }^{42}$ A. Maire, ${ }^{136}$ R. D. Majka, ${ }^{146}$ M. Malaev, ${ }^{96}$ Q. W. Malik, ${ }^{21}$ L. Malinina, ${ }^{74, b}$ D. Mal'Kevich, ${ }^{90}$ P. Malzacher, ${ }^{105}$ G. Mandaglio, ${ }^{55}$ V. Manko, ${ }^{86}$ F. Manso, ${ }^{134}$ V. Manzari, ${ }^{52}$

Y. Mao, ${ }^{6}$ M. Marchisone, ${ }^{135}$ J. Mareš, ${ }^{66}$ G. V. Margagliotti, ${ }^{25}$ A. Margotti, ${ }^{53}$ J. Margutti, ${ }^{63}$ A. Marín, ${ }^{105}$ C. Markert, ${ }^{119}$

M. Marquard, ${ }^{68}$ N. A. Martin, ${ }^{102}$ P. Martinengo, ${ }^{44}$ J. L. Martinez, ${ }^{125}$ M. I. Martínez, ${ }^{44}$ G. Martínez García, ${ }^{114}$

M. Martinez Pedreira, ${ }^{34}$ S. Masciocchi, ${ }^{105}$ M. Masera, ${ }^{26}$ A. Masoni, ${ }^{54}$ L. Massacrier, ${ }^{61}$ E. Masson, ${ }^{114}$ A. Mastroserio, ${ }^{52,138}$

A. M. Mathis, ${ }^{103,117}$ O. Matonoha, ${ }^{79}$ P. F. T. Matuoka, ${ }^{121}$ A. Matyja, ${ }^{118}$ C. Mayer, ${ }^{118}$ J. Mazer, ${ }^{130}$ M. Mazzilli, ${ }^{33}$

M. A. Mazzoni ${ }^{57}$ A. F. Mechler, ${ }^{68}$ F. Meddi, ${ }^{23}$ Y. Melikyan, ${ }^{62,91}$ A. Menchaca-Rocha, ${ }^{71}$ C. Mengke, ${ }^{6}$ E. Meninno, ${ }^{30,113}$

M. Meres, ${ }^{14}$ S. Mhlanga, ${ }^{124}$ Y. Miake, ${ }^{133}$ L. Micheletti, ${ }^{26}$ D. L. Mihaylov, ${ }^{103}$ K. Mikhaylov, ${ }^{74,90}$ A. Mischke, ${ }^{63, c}$

A. N. Mishra, ${ }^{69}$ D. Miśkowiec, ${ }^{105}$ A. Modak, ${ }^{3}$ N. Mohammadi,${ }^{34}$ A. P. Mohanty, ${ }^{63}$ B. Mohanty, ${ }^{84}$ M. Mohisin Khan, ${ }^{17, d}$

C. Mordasini, ${ }^{103}$ D. A. Moreira De Godoy, ${ }^{144}$ L. A. P. Moreno, ${ }^{44}$ I. Morozov, ${ }^{62}$ A. Morsch, ${ }^{34}$ T. Mrnjavac, ${ }^{34}$ V. Muccifora, ${ }^{51}$

E. Mudnic, ${ }^{35}$ D. Mühlheim, ${ }^{144}$ S. Muhuri, ${ }^{141}$ J. D. Mulligan, ${ }^{78}$ M. G. Munhoz, ${ }^{121}$ K. Münning, ${ }^{42}$ R. H. Munzer, ${ }^{68}$

H. Murakami, ${ }^{132}$ S. Murray, ${ }^{124}$ L. Musa, ${ }^{34}$ J. Musinsky, ${ }^{64}$ C. J. Myers, ${ }^{125}$ J. W. Myrcha, ${ }^{142}$ B. Naik, ${ }^{48}$ R. Nair, ${ }^{83}$ B. K. Nandi, ${ }^{48}$ R. Nania, ${ }^{10,53}$ E. Nappi, ${ }^{52}$ M. U. Naru, ${ }^{15}$ A. F. Nassirpour, ${ }^{79}$ C. Nattrass, ${ }^{130}$ R. Nayak, ${ }^{48}$ T. K. Nayak, ${ }^{84}$ S. Nazarenko, ${ }^{107}$ A. Neagu, ${ }^{21}$ R. A. Negrao De Oliveira, ${ }^{68}$ L. Nellen, ${ }^{69}$ S. V. Nesbo, ${ }^{36}$ G. Neskovic, ${ }^{39}$ D. Nesterov, ${ }^{112}$ L. T. Neumann, ${ }^{142}$ B. S. Nielsen, ${ }^{87}$ S. Nikolaev, ${ }^{86}$ S. Nikulin, ${ }^{86}$ V. Nikulin, ${ }^{96}$ F. Noferini, ${ }^{10,53}$ P. Nomokonov, ${ }^{74}$ J. Norman, ${ }^{77}$ N. Novitzky, ${ }^{133}$ P. Nowakowski, ${ }^{142}$ A. Nyanin, ${ }^{86}$ J. Nystrand, ${ }^{22}$ M. Ogino, ${ }^{80}$ A. Ohlson, ${ }^{79,102}$ J. Oleniacz, ${ }^{142}$ A. C. Oliveira Da Silva, ${ }^{121,130}$

M. H. Oliver, ${ }^{146}$ C. Oppedisano, ${ }^{58}$ R. Orava, ${ }^{43}$ A. Ortiz Velasquez, ${ }^{69}$ A. Oskarsson, ${ }^{79}$ J. Otwinowski, ${ }^{118} \mathrm{~K}$. Oyama, ${ }^{80}$ Y. Pachmayer, ${ }^{102}$ V. Pacik, ${ }^{87}$ D. Pagano, ${ }^{140}$ G. Paić,${ }^{69}$ J. Pan, ${ }^{143}$ A. K. Pandey, ${ }^{48}$ S. Panebianco, ${ }^{137}$ P. Pareek, ${ }^{49,141}$ J. Park, ${ }^{60}$ J. E. Parkkila, ${ }^{126}$ S. Parmar, ${ }^{98}$ S. P. Pathak, ${ }^{125}$ R. N. Patra, ${ }^{141}$ B. Paul, ${ }^{24,58}$ H. Pei, ${ }^{6}$ T. Peitzmann, ${ }^{63}$ X. Peng, ${ }^{6}$ L. G. Pereira, ${ }^{70}$ H. Pereira Da Costa, ${ }^{137}$ D. Peresunko, ${ }^{86}$ G. M. Perez, ${ }^{8}$ E. Perez Lezama, ${ }^{68}$ V. Peskov, ${ }^{68}$ Y. Pestov, ${ }^{4}$ V. Petráček, ${ }^{37}$ M. Petrovici, ${ }^{47}$ R. P. Pezzi, ${ }^{70}$ S. Piano, ${ }^{59}$ M. Pikna, ${ }^{14}$ P. Pillot, ${ }^{114}$ L. O. D. L. Pimentel, ${ }^{87}$ O. Pinazza, ${ }^{34,53}$ L. Pinsky, ${ }^{125}$ C. Pinto, ${ }^{28}$ S. Pisano, ${ }^{10,51}$ D. Pistone, ${ }^{55}$ M. Płoskoń, ${ }^{78}$ M. Planinic, ${ }^{97}$ F. Pliquett, ${ }^{68}$ J. Pluta, ${ }^{142}$ S. Pochybova, ${ }^{145, c}$

M. G. Poghosyan, ${ }^{94}$ B. Polichtchouk, ${ }^{89}$ N. Poljak, ${ }^{97}$ A. Pop, ${ }^{47}$ H. Poppenborg, ${ }^{144}$ S. Porteboeuf-Houssais, ${ }^{134}$ V. Pozdniakov ${ }^{74}$ S. K. Prasad, ${ }^{3}$ R. Preghenella, ${ }^{53}$ F. Prino, ${ }^{58}$ C. A. Pruneau, ${ }^{143}$ I. Pshenichnov, ${ }^{62}$ M. Puccio, ${ }^{26,34}$ V. Punin, ${ }^{107}$ J. Putschke, ${ }^{143}$

R. E. Quishpe, ${ }^{125}$ S. Ragoni, ${ }^{109}$ S. Raha, ${ }^{3}$ S. Rajput, ${ }^{99}$ J. Rak, ${ }^{126}$ A. Rakotozafindrabe, ${ }^{137}$ L. Ramello, ${ }^{32}$ F. Rami, ${ }^{136}$

R. Raniwala, ${ }^{100}$ S. Raniwala, ${ }^{100}$ S. S. Räsänen, ${ }^{43}$ R. Rath, ${ }^{49}$ V. Ratza, ${ }^{42}$ I. Ravasenga, ${ }^{31}$ K. F. Read, ${ }^{94,130}$ K. Redlich, ${ }^{83, e}$ A. Rehman, ${ }^{22}$ P. Reichelt, ${ }^{68}$ F. Reidt, ${ }^{34}$ X. Ren, ${ }^{6}$ R. Renfordt, ${ }^{68}$ Z. Rescakova, ${ }^{38}$ J.-P. Revol, ${ }^{10}$ K. Reygers, ${ }^{102}$ V. Riabov, ${ }^{96}$ T. Richert, ${ }^{79,87}$ M. Richter, ${ }^{21}$ P. Riedler, ${ }^{34}$ W. Riegler, ${ }^{34}$ F. Riggi, ${ }^{28}$ C. Ristea, ${ }^{67}$ S. P. Rode, ${ }^{49}$ M. Rodríguez Cahuantzi, ${ }^{44}$ K. Røed, ${ }^{21}$ R. Rogalev, ${ }^{89}$ E. Rogochaya, ${ }^{74}$ D. Rohr, ${ }^{34}$ D. Röhrich, ${ }^{22}$ P. S. Rokita, ${ }^{142}$ F. Ronchetti, ${ }^{51}$ E. D. Rosas, ${ }^{69}$ K. Roslon, ${ }^{142}$ A. Rossi, ${ }^{29,56}$ A. Rotondi, ${ }^{139}$ F. Roukoutakis, ${ }^{82}$ A. Roy, ${ }^{49}$ P. Roy, ${ }^{108}$ O. V. Rueda, ${ }^{79}$ R. Rui, ${ }^{25}$ B. Rumyantsev, ${ }^{74}$ A. Rustamov, ${ }^{85}$ E. Ryabinkin, ${ }^{86}$ Y. Ryabov, ${ }^{96}$ A. Rybicki, ${ }^{118}$ H. Rytkonen, ${ }^{126}$ S. Sadhu, ${ }^{141}$ S. Sadovsky, ${ }^{89}$ K. Šafařík, ${ }^{34,37}$ S. K. Saha, ${ }^{141}$

B. Sahoo, ${ }^{48}$ P. Sahoo, ${ }^{48,49}$ R. Sahoo, ${ }^{49}$ S. Sahoo, ${ }^{65}$ P. K. Sahu, ${ }^{65}$ J. Saini, ${ }^{141}$ S. Sakai, ${ }^{133}$ S. Sambyal, ${ }^{99}$ V. Samsonov, ${ }^{91,96}$

D. Sarkar, ${ }^{143}$ N. Sarkar, ${ }^{141}$ P. Sarma, ${ }^{41}$ V. M. Sarti, ${ }^{103}$ M. H. P. Sas, ${ }^{63}$ E. Scapparone, ${ }^{53}$ B. Schaefer, ${ }^{94}$ J. Schambach, ${ }^{119}$

H. S. Scheid, ${ }^{68}$ C. Schiaua, ${ }^{47}$ R. Schicker, ${ }^{102}$ A. Schmah, ${ }^{102}$ C. Schmidt, ${ }^{105}$ H. R. Schmidt, ${ }^{101}$ M. O. Schmidt, ${ }^{102}$

M. Schmidt, ${ }^{101}$ N. V. Schmidt, ${ }^{68,94}$ A. R. Schmier, ${ }^{130}$ J. Schukraft, ${ }^{87}$ Y. Schutz, ${ }^{34,136}$ K. Schwarz, ${ }^{105}$ K. Schweda, ${ }^{105}$ G. Scioli, ${ }^{27}$

E. Scomparin, ${ }^{58}$ M. Šefč́́k, ${ }^{38}$ J. E. Seger, ${ }^{16}$ Y. Sekiguchi, ${ }^{132}$ D. Sekihata, ${ }^{45,132}$ I. Selyuzhenkov, ${ }^{91,105}$ S. Senyukov, ${ }^{136}$

D. Serebryakov, ${ }^{62}$ E. Serradilla, ${ }^{71}$ A. Sevcenco, ${ }^{67}$ A. Shabanov, ${ }^{62}$ A. Shabetai, ${ }^{114}$ R. Shahoyan, ${ }^{34}$ W. Shaikh, ${ }^{108}$

A. Shangaraev, ${ }^{89}$ A. Sharma, ${ }^{98}$ A. Sharma, ${ }^{99}$ H. Sharma, ${ }^{118}$ M. Sharma, ${ }^{99}$ N. Sharma, ${ }^{98}$ A. I. Sheikh, ${ }^{141}$ K. Shigaki, ${ }^{45}$

M. Shimomura, ${ }^{81}$ S. Shirinkin, ${ }^{90}$ Q. Shou, ${ }^{111}$ Y. Sibiriak, ${ }^{86}$ S. Siddhanta, ${ }^{54}$ T. Siemiarczuk, ${ }^{83}$ D. Silvermyr,${ }^{79}$ G. Simatovic, ${ }^{88}$

G. Simonetti, ${ }^{34,103}$ R. Singh, ${ }^{84}$ R. Singh, ${ }^{99}$ R. Singh, ${ }^{49}$ V. K. Singh, ${ }^{141}$ V. Singhal, ${ }^{141}$ T. Sinha, ${ }^{108}$ B. Sitar, ${ }^{14}$ M. Sitta, ${ }^{32}$

T. B. Skaali, ${ }^{21}$ M. Slupecki, ${ }^{126}$ N. Smirnov, ${ }^{146}$ R. J. M. Snellings, ${ }^{63}$ T. W. Snellman,,${ }^{43,126}$ C. Soncco, ${ }^{110}$ J. Song, ${ }^{60,125}$

A. Songmoolnak, ${ }^{115}$ F. Soramel, ${ }^{29}$ S. Sorensen, ${ }^{130}$ I. Sputowska, ${ }^{118}$ J. Stachel, ${ }^{102}$ I. Stan, ${ }^{67}$ P. Stankus, ${ }^{94}$ P. J. Steffanic, ${ }^{130}$

E. Stenlund, ${ }^{79}$ D. Stocco, ${ }^{114}$ M. M. Storetvedt,${ }^{36}$ L. D. Stritto, ${ }^{30}$ A. A. P. Suaide, ${ }^{121}$ T. Sugitate, ${ }^{45}$ C. Suire, ${ }^{61}$ M. Suleymanov, ${ }^{15}$

M. Suljic, ${ }^{34}$ R. Sultanov, ${ }^{90}$ M. Šumbera, ${ }^{93}$ S. Sumowidagdo, ${ }^{50}$ S. Swain, ${ }^{65}$ A. Szabo, ${ }^{14}$ I. Szarka, ${ }^{14}$ U. Tabassam, ${ }^{15}$

G. Taillepied, ${ }^{134}$ J. Takahashi, ${ }^{122}$ G. J. Tambave, ${ }^{22}$ S. Tang, ${ }^{6,134}$ M. Tarhini, ${ }^{114}$ M. G. Tarzila, ${ }^{47}$ A. Tauro, ${ }^{34}$ G. Tejeda Muñoz, ${ }^{44}$

A. Telesca, ${ }^{34}$ C. Terrevoli, ${ }^{125}$ D. Thakur, ${ }^{49}$ S. Thakur, ${ }^{141}$ D. Thomas, ${ }^{119}$ F. Thoresen, ${ }^{87}$ R. Tieulent, ${ }^{135}$ A. Tikhonov, ${ }^{62}$

A. R. Timmins, ${ }^{125}$ A. Toia, ${ }^{68}$ N. Topilskaya, ${ }^{62}$ M. Toppi,${ }^{51}$ F. Torales-Acosta, ${ }^{20} \mathrm{~S}$. R. Torres, ${ }^{9,120}$ A. Trifiro, ${ }^{55} \mathrm{~S}$. Tripathy, ${ }^{49}$ T. Tripathy, ${ }^{48}$ S. Trogolo ${ }^{29}$ G. Trombetta, ${ }^{33}$ L. Tropp, ${ }^{38}$ V. Trubnikov, ${ }^{2}$ W. H. Trzaska, ${ }^{126}$ T. P. Trzcinski, ${ }^{142}$ B. A. Trzeciak, ${ }^{63}$ T. Tsuji, ${ }^{132}$ A. Tumkin, ${ }^{107}$ R. Turrisi, ${ }^{56}$ T. S. Tveter, ${ }^{21}$ K. Ullaland, ${ }^{22}$ E. N. Umaka, ${ }^{125}$ A. Uras, ${ }^{135}$ G. L. Usai, ${ }^{24}$ A. Utrobicic, ${ }^{97}$ M. Vala ${ }^{38}$ N. Valle, ${ }^{139}$ S. Vallero, ${ }^{58}$ N. van der Kolk, ${ }^{63}$ L. V. R. van Doremalen, ${ }^{63}$ M. van Leeuwen, ${ }^{63}$ P. Vande Vyvre, ${ }^{34}$ D. Varga, ${ }^{145}$ Z. Varga, ${ }^{145}$ M. Varga-Kofarago, ${ }^{145}$ A. Vargas, ${ }^{44}$ M. Vargyas, ${ }^{126}$ M. Vasileiou, ${ }^{82}$ A. Vasiliev, ${ }^{86}$

O. Vázquez Doce, ${ }^{103,117}$ V. Vechernin, ${ }^{112}$ A. M. Veen, ${ }^{63}$ E. Vercellin, ${ }^{26}$ S. Vergara Limón, ${ }^{44}$ L. Vermunt, ${ }^{63}$ R. Vernet, ${ }^{7}$

R. Vértesi, ${ }^{145}$ L. Vickovic, ${ }^{35}$ J. Viinikainen, ${ }^{126}$ Z. Vilakazi, ${ }^{131}$ O. Villalobos Baillie, ${ }^{109}$ A. Villatoro Tello, ${ }^{44} \mathrm{G}$. Vino, ${ }^{52}$

A. Vinogradov, ${ }^{86}$ T. Virgili, ${ }^{30}$ V. Vislavicius,${ }^{87}$ A. Vodopyanov, ${ }^{74}$ B. Volkel, ${ }^{34}$ M. A. Völkl, ${ }^{101}$ K. Voloshin, ${ }^{90}$ S. A. Voloshin, ${ }^{143}$ G. Volpe, ${ }^{33}$ B. von Haller, ${ }^{34}$ I. Vorobyev, ${ }^{103}$ D. Voscek, ${ }^{116}$ J. Vrláková, ${ }^{38}$ B. Wagner, ${ }^{22}$ M. Weber, ${ }^{113}$ S. G. Weber, ${ }^{105,144}$ A. Wegrzynek, ${ }^{34}$ D. F. Weiser, ${ }^{102}$ S. C. Wenzel, ${ }^{34}$ J. P. Wessels,${ }^{144}$ J. Wiechula, ${ }^{68}$ J. Wikne, ${ }^{21}$ G. Wilk, ${ }^{83}$ J. Wilkinson, ${ }^{10,53}$ 
G. A. Willems ${ }^{34}$ E. Willsher, ${ }^{109}$ B. Windelband, ${ }^{102}$ W. E. Witt, ${ }^{130}$ Y. Wu, ${ }^{128}$ R. Xu,${ }^{6}$ S. Yalcin, ${ }^{76}$ K. Yamakawa, ${ }^{45}$ S. Yang ${ }^{22}$ S. Yano, ${ }^{137}$ Z. Yin, ${ }^{6}$ H. Yokoyama ${ }^{63}$ I.-K. Yoo, ${ }^{18}$ J. H. Yoon ${ }^{60}$ S. Yuan, ${ }^{22}$ A. Yuncu, ${ }^{102}$ V. Yurchenko, ${ }^{2}$ V. Zaccolo, ${ }^{25}$ A. Zaman, ${ }^{15}$ C. Zampolli, ${ }^{34}$ H. J. C. Zanoli, ${ }^{63,121}$ N. Zardoshti, ${ }^{34}$ A. Zarochentsev, ${ }^{112}$ P. Závada, ${ }^{66}$ N. Zaviyalov, ${ }^{107}$ H. Zbroszczyk, ${ }^{142}$ M. Zhalov, ${ }^{96}$ S. Zhang, ${ }^{111}$ X. Zhang, ${ }^{6}$ Z. Zhang, ${ }^{6}$ V. Zherebchevskii, ${ }^{12}$ N. Zhigareva, ${ }^{90}$ D. Zhou, ${ }^{6}$ Y. Zhou, ${ }^{87}$ Z. Zhou, ${ }^{22}$ J. Zhu, ${ }^{6,105}$ Y. Zhu, ${ }^{6}$ A. Zichichi, ${ }^{10,27}$ M. B. Zimmermann, ${ }^{34}$ G. Zinovjev, ${ }^{2}$ and N. Zurlo ${ }^{140}$

\section{(ALICE Collaboration)}

${ }^{1}$ A.I. Alikhanyan National Science Laboratory (Yerevan Physics Institute) Foundation, Yerevan, Armenia

${ }^{2}$ Bogolyubov Institute for Theoretical Physics, National Academy of Sciences of Ukraine, Kiev, Ukraine

${ }^{3}$ Bose Institute, Department of Physics and Centre for Astroparticle Physics and Space Science (CAPSS), Kolkata, India

${ }^{4}$ Budker Institute for Nuclear Physics, Novosibirsk, Russia

${ }^{5}$ California Polytechnic State University, San Luis Obispo, California, United States

${ }^{6}$ Central China Normal University, Wuhan, China

${ }^{7}$ Centre de Calcul de l'IN2P3, Villeurbanne, Lyon, France

${ }^{8}$ Centro de Aplicaciones Tecnológicas y Desarrollo Nuclear (CEADEN), Havana, Cuba

${ }^{9}$ Centro de Investigación y de Estudios Avanzados (CINVESTAV), Mexico City and Mérida, Mexico

${ }^{10}$ Centro Fermi - Museo Storico della Fisica e Centro Studi e Ricerche "Enrico Fermi”, Rome, Italy

${ }^{11}$ Chicago State University, Chicago, Illinois, United States

${ }^{12}$ China Institute of Atomic Energy, Beijing, China

${ }^{13}$ Chonbuk National University, Jeonju, Republic of Korea

${ }^{14}$ Comenius University Bratislava, Faculty of Mathematics, Physics and Informatics, Bratislava, Slovakia

${ }^{15}$ COMSATS University Islamabad, Islamabad, Pakistan

${ }^{16}$ Creighton University, Omaha, Nebraska, United States

${ }^{17}$ Department of Physics, Aligarh Muslim University, Aligarh, India

${ }^{18}$ Department of Physics, Pusan National University, Pusan, Republic of Korea

${ }^{19}$ Department of Physics, Sejong University, Seoul, Republic of Korea

${ }^{20}$ Department of Physics, University of California, Berkeley, California, United States

${ }^{21}$ Department of Physics, University of Oslo, Oslo, Norway

${ }^{22}$ Department of Physics and Technology, University of Bergen, Bergen, Norway

${ }^{23}$ Dipartimento di Fisica dell'Università 'La Sapienza' and Sezione INFN, Rome, Italy

${ }^{24}$ Dipartimento di Fisica dell'Università and Sezione INFN, Cagliari, Italy

${ }^{25}$ Dipartimento di Fisica dell'Università and Sezione INFN, Trieste, Italy

${ }^{26}$ Dipartimento di Fisica dell'Università and Sezione INFN, Turin, Italy

${ }^{27}$ Dipartimento di Fisica e Astronomia dell'Università and Sezione INFN, Bologna, Italy

${ }^{28}$ Dipartimento di Fisica e Astronomia dell'Università and Sezione INFN, Catania, Italy

${ }^{29}$ Dipartimento di Fisica e Astronomia dell'Università and Sezione INFN, Padova, Italy

${ }^{30}$ Dipartimento di Fisica 'E.R. Caianiello' dell'Università and Gruppo Collegato INFN, Salerno, Italy

${ }^{31}$ Dipartimento DISAT del Politecnico and Sezione INFN, Turin, Italy

${ }^{32}$ Dipartimento di Scienze e Innovazione Tecnologica dell'Università del Piemonte Orientale and INFN Sezione di Torino, Alessandria, Italy

${ }^{33}$ Dipartimento Interateneo di Fisica 'M. Merlin' and Sezione INFN, Bari, Italy

${ }^{34}$ European Organization for Nuclear Research (CERN), Geneva, Switzerland

${ }^{35}$ Faculty of Electrical Engineering, Mechanical Engineering and Naval Architecture, University of Split, Split, Croatia

${ }^{36}$ Faculty of Engineering and Science, Western Norway University of Applied Sciences, Bergen, Norway

${ }^{37}$ Faculty of Nuclear Sciences and Physical Engineering, Czech Technical University in Prague, Prague, Czech Republic

${ }^{38}$ Faculty of Science, P.J. Šafárik University, Košice, Slovakia

${ }^{39}$ Frankfurt Institute for Advanced Studies, Johann Wolfgang Goethe-Universität Frankfurt, Frankfurt, Germany

${ }^{40}$ Gangneung-Wonju National University, Gangneung, Republic of Korea

${ }^{41}$ Gauhati University, Department of Physics, Guwahati, India

${ }^{42}$ Helmholtz-Institut für Strahlen- und Kernphysik, Rheinische Friedrich-Wilhelms-Universität Bonn, Bonn, Germany

${ }^{43}$ Helsinki Institute of Physics (HIP), Helsinki, Finland

${ }^{44}$ High Energy Physics Group, Universidad Autónoma de Puebla, Puebla, Mexico

${ }^{45}$ Hiroshima University, Hiroshima, Japan

${ }^{46}$ Hochschule Worms, Zentrum für Technologietransfer und Telekommunikation (ZTT), Worms, Germany

${ }^{47}$ Horia Hulubei National Institute of Physics and Nuclear Engineering, Bucharest, Romania

${ }^{48}$ Indian Institute of Technology Bombay (IIT), Mumbai, India

${ }^{49}$ Indian Institute of Technology Indore, Indore, India

${ }^{50}$ Indonesian Institute of Sciences, Jakarta, Indonesia 
${ }^{51}$ INFN, Laboratori Nazionali di Frascati, Frascati, Italy

${ }^{52}$ INFN, Sezione di Bari, Bari, Italy

${ }^{53}$ INFN, Sezione di Bologna, Bologna, Italy

${ }^{54}$ INFN, Sezione di Cagliari, Cagliari, Italy

${ }^{55}$ INFN, Sezione di Catania, Catania, Italy

${ }^{56}$ INFN, Sezione di Padova, Padova, Italy

${ }^{57}$ INFN, Sezione di Roma, Rome, Italy

${ }^{58}$ INFN, Sezione di Torino, Turin, Italy

${ }^{59}$ INFN, Sezione di Trieste, Trieste, Italy

${ }^{60}$ Inha University, Incheon, Republic of Korea

${ }^{61}$ Institut de Physique Nucléaire d'Orsay (IPNO), Institut National de Physique Nucléaire et de Physique des Particules (IN2P3/CNRS), Université de Paris-Sud, Université Paris-Saclay, Orsay, France

${ }^{62}$ Institute for Nuclear Research, Academy of Sciences, Moscow, Russia

${ }^{63}$ Institute for Subatomic Physics, Utrecht University/Nikhef, Utrecht, Netherlands

${ }^{64}$ Institute of Experimental Physics, Slovak Academy of Sciences, Košice, Slovakia

${ }^{65}$ Institute of Physics, Homi Bhabha National Institute, Bhubaneswar, India

${ }^{66}$ Institute of Physics of the Czech Academy of Sciences, Prague, Czech Republic

${ }^{67}$ Institute of Space Science (ISS), Bucharest, Romania

${ }^{68}$ Institut für Kernphysik, Johann Wolfgang Goethe-Universität Frankfurt, Frankfurt, Germany

${ }^{69}$ Instituto de Ciencias Nucleares, Universidad Nacional Autónoma de México, Mexico City, Mexico

${ }^{70}$ Instituto de Física, Universidade Federal do Rio Grande do Sul (UFRGS), Porto Alegre, Brazil

${ }^{71}$ Instituto de Física, Universidad Nacional Autónoma de México, Mexico City, Mexico

${ }^{72}$ iThemba LABS, National Research Foundation, Somerset West, South Africa

${ }^{73}$ Johann-Wolfgang-Goethe Universität Frankfurt Institut für Informatik, Fachbereich Informatik und Mathematik, Frankfurt, Germany

${ }^{74}$ Joint Institute for Nuclear Research (JINR), Dubna, Russia

${ }^{75}$ Korea Institute of Science and Technology Information, Daejeon, Republic of Korea

${ }^{76}$ KTO Karatay University, Konya, Turkey

${ }^{77}$ Laboratoire de Physique Subatomique et de Cosmologie, Université Grenoble-Alpes, CNRS-IN2P3, Grenoble, France

${ }^{78}$ Lawrence Berkeley National Laboratory, Berkeley, California, United States

${ }^{79}$ Lund University Department of Physics, Division of Particle Physics, Lund, Sweden

${ }^{80}$ Nagasaki Institute of Applied Science, Nagasaki, Japan

${ }^{81}$ Nara Women's University (NWU), Nara, Japan

${ }^{82}$ National and Kapodistrian University of Athens, School of Science, Department of Physics, Athens, Greece

${ }^{83}$ National Centre for Nuclear Research, Warsaw, Poland

${ }^{84}$ National Institute of Science Education and Research, Homi Bhabha National Institute, Jatni, India

${ }^{85}$ National Nuclear Research Center, Baku, Azerbaijan

${ }^{86}$ National Research Centre Kurchatov Institute, Moscow, Russia

${ }^{87}$ Niels Bohr Institute, University of Copenhagen, Copenhagen, Denmark

${ }^{88}$ Nikhef, National institute for subatomic physics, Amsterdam, Netherlands

${ }^{89}$ NRC Kurchatov Institute IHEP, Protvino, Russia

${ }^{90}$ NRC Kurchatov Institute - ITEP, Moscow, Russia

${ }^{91}$ NRNU Moscow Engineering Physics Institute, Moscow, Russia

${ }^{92}$ Nuclear Physics Group, STFC Daresbury Laboratory, Daresbury, United Kingdom

${ }^{93}$ Nuclear Physics Institute of the Czech Academy of Sciences, Řež u Prahy, Czech Republic

${ }^{94}$ Oak Ridge National Laboratory, Oak Ridge, Tennessee, United States

${ }^{95}$ Ohio State University, Columbus, Ohio, United States

${ }^{96}$ Petersburg Nuclear Physics Institute, Gatchina, Russia

${ }^{97}$ Physics department, Faculty of science, University of Zagreb, Zagreb, Croatia

${ }^{98}$ Physics Department, Panjab University, Chandigarh, India

${ }^{99}$ Physics Department, University of Jammu, Jammu, India

${ }^{100}$ Physics Department, University of Rajasthan, Jaipur, India

${ }^{101}$ Physikalisches Institut, Eberhard-Karls-Universität Tübingen, Tübingen, Germany

${ }^{102}$ Physikalisches Institut, Ruprecht-Karls-Universität Heidelberg, Heidelberg, Germany

${ }^{103}$ Physik Department, Technische Universität München, Munich, Germany ${ }^{104}$ Politecnico di Bari, Bari, Italy

${ }^{105}$ Research Division and ExtreMe Matter Institute EMMI, GSI Helmholtzzentrum für Schwerionenforschung GmbH, Darmstadt, Germany

${ }^{106}$ Rudjer Bošković Institute, Zagreb, Croatia

${ }^{107}$ Russian Federal Nuclear Center (VNIIEF), Sarov, Russia

${ }^{108}$ Saha Institute of Nuclear Physics, Homi Bhabha National Institute, Kolkata, India 


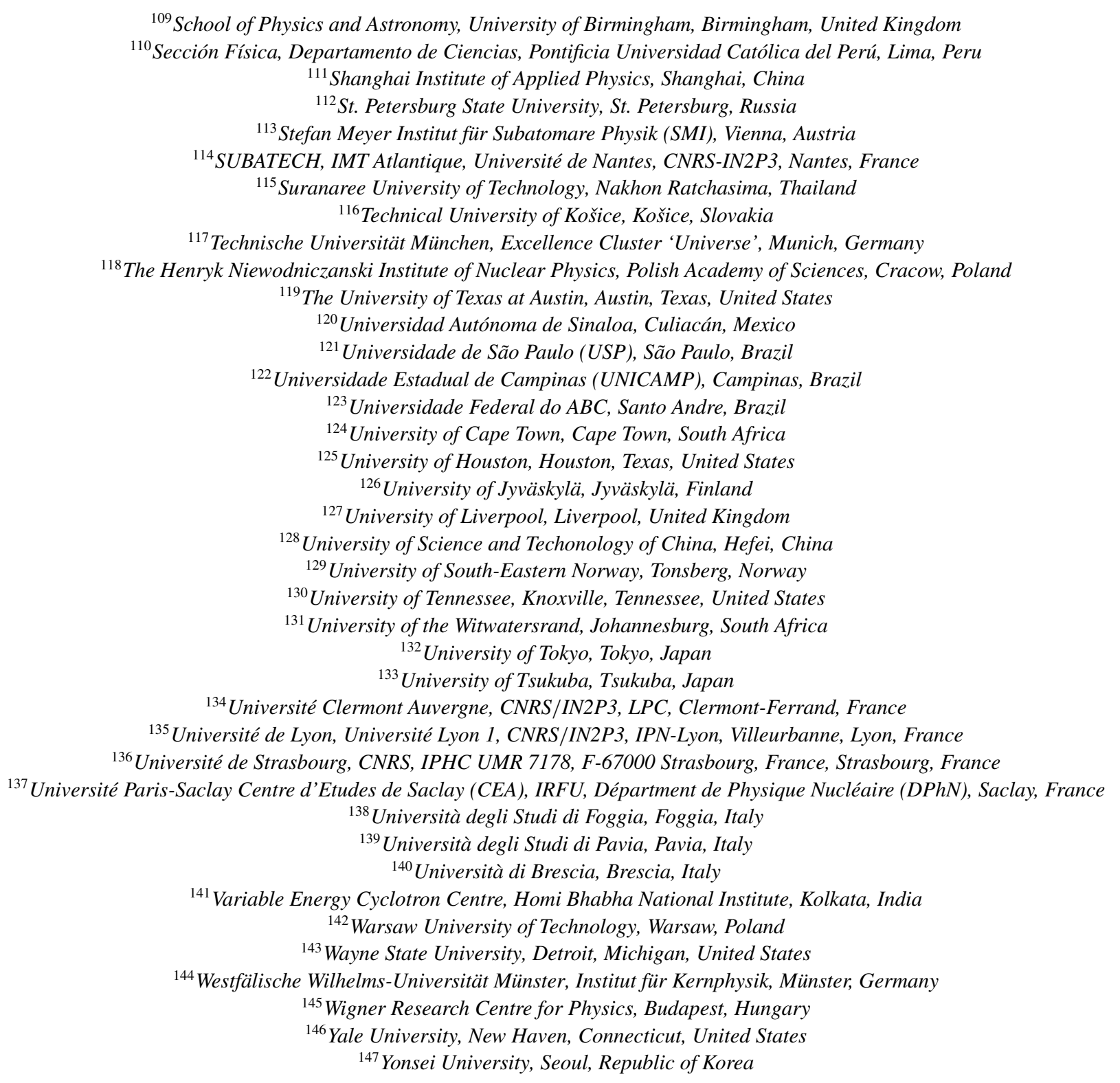

${ }^{\text {a}}$ Dipartimento DET del Politecnico di Torino, Turin, Italy.

${ }^{\mathrm{b}}$ M.V. Lomonosov Moscow State University, D.V. Skobeltsyn Institute of Nuclear, Physics, Moscow, Russia.

${ }^{\mathrm{c}}$ Deceased.

${ }^{\mathrm{d}}$ Department of Applied Physics, Aligarh Muslim University, Aligarh, India.

${ }^{\mathrm{e}}$ Institute of Theoretical Physics, University of Wroclaw, Poland. 